\title{
A VANISHING THEOREM FOR CHARACTERISTIC CLASSES OF ODD-DIMENSIONAL MANIFOLD BUNDLES
}

\author{
JOHANNES EBERT
}

\begin{abstract}
We show how the Atiyah-Singer family index theorem for both, usual and self-adjoint elliptic operators fits naturally into the framework of the MadsenTillmann spectra. Our main theorem concerns bundles of odd-dimensional manifolds. Using completely functional-analytic methods, we show that for any smooth proper oriented fibre bundle $E \rightarrow X$ with odd-dimensional fibres, the family index $\operatorname{ind}(B) \in K^{1}(X)$ of the odd signature operator is trivial. The Atiyah-Singer theorem allows us to draw a topological conclusion: the generalized Madsen-Tillmann map $\alpha: B \operatorname{Diff}^{+}\left(M^{2 m-1}\right) \rightarrow \Omega^{\infty} \operatorname{MTSO}(2 m-1)$ kills the Hirzebruch $\mathcal{L}$-class in rational cohomology. If $m=2$, this means that $\alpha$ induces the zero map in rational cohomology. In particular, the three-dimensional analogue of the Madsen-Weiss theorem is wrong. In higher dimensions, we show that the Hirzebruch $\mathcal{L}$-class is the only class for which such a vanishing theorem holds. For 3-manifolds $M$, we also prove the triviality of $\alpha: B \operatorname{Diff}^{+}(M) \rightarrow \operatorname{MTSO}(3)$ in mod $p$ cohomology in many cases.
\end{abstract}

\section{Contents}

1. Introduction and statement of results

1

2. Background material on Madsen-Tillmann spectra 4

3. Background material on index theory 13

4. The index theorem for the odd signature operator 16

5. Nonvanishing result for other generalized MMM-classes 21

6. A real refinement and the one-dimensional case 21

7. Vanishing theorems in mod $p$ cohomology and an open problem 23

References 26

\section{Introduction AND STATEMENT OF RESUlts}

One of the greatest achievements of algebraic topology in the last decade are the two proofs of Mumford's conjecture on the homology of the stable mapping class group by Madsen and Weiss [25] and by Galatius, Madsen, Tillmann and Weiss [17. The Pontrjagin-Thom construction is crucial for both proofs; it provides a map from the classifying space of the diffeomorphism group of a compact surface to the infinite loop space $\Omega^{\infty} \operatorname{MTSO}(2)$ of the Madsen-Tillmann spectrum, in other words the Thom spectrum of the inverse of the universal complex line bundle.

The proof in [17] consists of two parts. One part (essentially due to Tillmann [36]), exclusively applies to 2-dimensional manifolds, because it relies on two deep

1991 Mathematics Subject Classification. 55R40, 58J20, 58J20, 57R90. 
results of surface theory (the Harer-Ivanov homological stability theorem and the Earle-Eells theorem on the contractibility of the components of the diffeomorphism group of surfaces of negative Euler number). The other part of the proof, however, is valid for manifolds of arbitrary dimension and with general "tangential structures" and provides a vast generalization of the classical Pontrjagin-Thom theorem relating bordism theory of smooth manifolds and stable homotopy.

Given an oriented (we will ignore more general tangential structures throughout the present paper) closed manifold $M$ of dimension $n$, there exists a map

$$
\alpha_{E_{M}}: B \operatorname{Diff}^{+}(M) \rightarrow \Omega^{\infty} \operatorname{MTSO}(n),
$$

where $\operatorname{MTSO}(n)$ denotes the Thom spectrum of the inverse of the universal $n$ dimensional oriented vector bundle. Let $\mathrm{Cob}_{n}^{+}$be the oriented $n$-dimensional cobordism category: objects are closed $(n-1)$-dimensional manifolds, morphisms are oriented cobordisms and composition is given by gluing cobordisms. With a suitable topology on object and morphism spaces, $\mathrm{Cob}_{n}^{+}$becomes a topological category. The maps $\alpha$ from 1.0.1 assemble to a map

$$
\alpha^{G M T W}: \Omega B \mathrm{Cob}_{n}^{+} \rightarrow \Omega^{\infty} \operatorname{MTSO}(n),
$$

and the main result of [17] states that $\alpha^{G M T W}$ is a homotopy equivalence. Moreover, for any closed $n$-manifold $M$, there is a tautological map $\Phi_{M}: B \operatorname{Diff}^{+}(M) \rightarrow$ $\Omega B \mathrm{Cob}_{n}^{+}$and $\alpha^{G M T W} \circ \Phi_{M}=\alpha_{E_{M}}$.

The exclusive result for two-dimensional manifolds is that when $M$ is a closed connected oriented surface of genus $g$, then $\Phi_{M}$ induces an isomorphism on integral homology groups of degrees $* \leq g / 2-1$. Both theorems together provide an isomorphism of the homology of $B \operatorname{Diff}^{+}(M)$ and $\Omega^{\infty} \operatorname{MTSO}(2)$ (in that range of degrees).

In this paper, we study the map $\alpha_{E_{M}}$ (or, equivalently, $\Phi_{M}$ ) when $M$ is an oriented closed manifold of odd dimension. It turns out that $\alpha_{E_{M}}$ fails to be an isomorphism in homology in any range and that no clue about the homology of $B \operatorname{Diff}^{+}(M)$ can be derived from the study of $\alpha_{E_{M}}$. This seems to be an unsatisfactory state of affairs and therefore we attempt to arouse the reader's curiosity by the following remark:

Even if the map $\alpha$ fails to be an "equivalence" of some kind, it still contains interesting information about $B \operatorname{Diff}^{+}(M)$. Any cohomology class of $\Omega^{\infty} \operatorname{MTSO}(n)$ (in an arbitrary generalized cohomology theory) yields, via $\alpha_{E_{M}}$, a cohomology class of $B \operatorname{Diff}^{+}(M)$, also known as a characteristic class of smooth oriented $M$-bundles. One should think of these characteristic classes as "universal" classes in the sense that they are defined for all oriented $n$-manifolds and are defined using only the local structure of the manifold.

Examples are the generalized MMM classes

$$
f_{!}\left(c\left(T_{v} E\right)\right) \in H^{*-n}(B ; R),
$$


where $f: E \rightarrow B$ is a smooth oriented fibre bundle with vertical tangent bundle $T_{v} E, R$ is a ring and $c \in H^{*}(B S O(n) ; R)$ is a characteristic class of oriented vector bundles. The generalized MMM classes come from spectrum cohomology classes of $\operatorname{MTSO}(n)$.

Other examples come from index theory of elliptic operators. Any sufficiently natural elliptic differential operator on oriented $n$-manifolds defines a characteristic class in $K^{0}$ (namely, the family index). Likewise, a natural self-adjoint elliptic operator has a family index in $K^{-1}$ and so it defines a characteristic class in $K^{-1}$. An application of the Atiyah-Singer Index theorem shows that these index-theoretic classes also come from $\operatorname{MTSO}(n)$.

Index theory will be the central tool in our study of $\alpha_{E_{M}}$. On any closed oriented Riemannian manifold of odd dimension, there is the odd signature operator $D$ : $\mathcal{A}^{e v}(M) \rightarrow \mathcal{A}^{e v}(M)$ on forms of even degree. It is self-adjoint, elliptic and its kernel is the space of harmonic form of even degree, which is isomorphic to $H^{e v}(M ; \mathbb{C})$. Given any smooth oriented $M$-bundle $f: E \rightarrow B$ we can choose a Riemannian metric on the fibres and study the induced family of elliptic self-adjoint operators. Here is the central result of the present paper.

Theorem 1.0.2. The family index of the odd signature operator on an oriented bundle $E \rightarrow B$ with odd-dimensional fibres is trivial, $\operatorname{ind}(D)=0 \in K^{1}(B)$.

The proof of this result is entirely analytic; it is based on the fact that the kernel dimension of $D$ is constant. Therefore the Atiyah-Singer index theorem allows us to draw topological conclusions from Theorem 1.0.2. Here is one of them:

Theorem 1.0.3. For any closed oriented $2 m-1$-dimensional manifold $M$, the Madsen-Tillmann map $\Sigma^{\infty}\left(B \operatorname{Diff}^{+}(M)\right)_{+} \rightarrow \operatorname{MTSO}(2 m-1)$ kills the Hirzebruch $\mathcal{L}$-class th- $L_{2 m-1} \mathcal{L} \in H^{4 *-2 m+1}(\operatorname{MTSO}(2 m+1) ; \mathbb{Q})$.

In particular, for any oriented smooth fibre bundle $f: E \rightarrow B$ with fibre $M$, the generalized $M M M$ class $f_{!}\left(\mathcal{L}\left(T_{v} E\right)\right) \in H^{*}(B ; \mathbb{Q})$ is trivial.

The precise meaning of this theorem will be clarified in the main text. If $m=2$, Theorem 1.0 .3 implies:

Corollary 1.0.4. If $\operatorname{dim} M=3$, the Madsen-Tillmann map $\alpha: B \operatorname{Diff}^{+}(M) \rightarrow$ $\Omega^{\infty} \mathrm{MTSO}(3)$ is trivial in rational cohomology (in positive degrees).

This is an amusing result. Recently, Hatcher and Wahl [18] showed an analogue of the Harer-Ivanov homological stability for mapping class groups of 3-manifolds. Moreover, for large classes 3-dimensional manifolds, it is known that the components of the diffeomorphism group are contractible (but that tends to become wrong after stabilization). One might be tempted to think that these results helps to make the proof of the analogue of the Mumford conjecture valid, leading to a description of the stable homology of mapping class groups of 3-manifolds in terms of the homology of $\Omega^{\infty} \operatorname{MTSO}(3)$. Corollary 1.0 .4 shows that this is not the case.

Theorem 1.0.2 has also consequences in $\bmod p$-cohomology. We prove two things for oriented 3-manifolds in that direction. Fix an oriented 3-manifold $M$. We shall prove: 
- Fix $k \geq 1$. Then for almost all odd primes $p$, the map $\alpha^{*}: H^{4 k-1}\left(\operatorname{MTSO}(3) ; \mathbb{F}_{p}\right) \rightarrow$ $H^{4 k-1}\left(B \operatorname{Diff}^{+}(M) ; \mathbb{F}_{p}\right)$ is zero (Theorem 7.0.6).

- Fix an odd prime $p$. Then $\alpha^{*}: H^{4 k-1}\left(\operatorname{MTSO}(3) ; \mathbb{F}_{p}\right) \rightarrow H^{4 k-1}\left(B \operatorname{Diff}^{+}(M) ; \mathbb{F}_{p}\right)$ is zero for an infinite number of values for $k$ (Theorem 7.0.7).

In both cases, the primes to which the theorem applies does not depend on $M$.

We also prove that in higher odd dimensions, the map $\alpha$ is usually nontrivial in rational cohomology (Theorem 5.0.4). More precisely, classes which are not multiples of the Hirzbruch $\mathcal{L}$-class are in general not in the kernel of $\alpha^{*}$.

In dimensions of the form $4 r+1$, there is a real refinement of Theorem 1.0.2, More precisely, the odd signature operator has an index in real K-theory. This real index, however, is usually not zero.

1.1. Outline of the paper. Section 2 is a survey on the stable homotopy theory which is needed in this paper. We briefly discuss general Thom spectra, the MadsenTillmann spectra, the Pontrjagin-Thom construction, the Madsen-Tillmann map and Thom isomorphisms. Subsection 2.4 is devoted to a study of the component group $\pi_{0}(\operatorname{MTSO}(n))$. This is needed later in section 6. Section 3 provides the necessary constructions from index theory. In section 4, we discuss the odd signature operator and prove Theorem 1.0.2. Also, we show 1.0.3 and 1.0.4. In section 5, we discuss that the nonvanishing result mentioned above. Section 6 discusses the real index of the odd signature operator. Finally, in section 7 , we discuss the vanishing theorem in finite characteristic.

1.2. Acknowledgements. The author is indebted to a number of people for enlightening discussions about the mathematics in this paper. Among them are Oscar Randal-Williams, Ulrike Tillmann, Ib Madsen and Ulrich Bunke. Last but not least, I have to acknowledge the hospitality of the Mathematical Institute of the University of Oxford and the financial support from the Postdoctoral program of the German Academic Exchange Service (DAAD) which I enjoyed when this project was begun.

\section{BaCKGround material on MAdSEn-Tillmann SPECTRA}

Before we start this survey, let us fix the convention that all vector bundles are endowed with metrics (euclidean or hermitian, depending on the context).

2.1. Stable vector bundles and their Thom spectra. A stable vector bundle on a space $X$ is a pair $\left(V_{0}, V_{1}\right)$ of real vector bundles on $X$; one should think of it as the formal difference $V_{0}-V_{1}$, and we will sometimes use this more suggestive notation. The rank of $\left(V_{0}, V_{1}\right)$ is the difference $\operatorname{dim} V_{0}-\operatorname{dim} V_{1}$. An isomorphism $\left(V_{0}, V_{1}\right) \rightarrow\left(U_{0}, U_{1}\right)$ is represented by a pair $(W, \theta)$ where $W$ is a vector bundle and

$$
\theta: V_{0} \oplus U_{1} \oplus W \rightarrow V_{1} \oplus U_{0} \oplus W
$$

is a bundle isomorphism. Two pairs $(\theta, W),\left(\theta^{\prime}, W^{\prime}\right)$ represent the same morphism if there exists a vector bundle $W^{\prime \prime}$ and an isomorphism $\varphi: W^{\prime} \cong W \oplus W^{\prime \prime}$ such that $\theta^{\prime}=\varphi^{-1} \circ\left(\theta \oplus \operatorname{id}_{W^{\prime \prime}}\right) \circ \varphi$ (and then take the equivalence relation that this generates). 
The composition of $\theta: V_{0} \oplus U_{1} \oplus W \rightarrow V_{1} \oplus U_{0} \oplus W$ and $\phi: V_{0} \oplus S_{1} \oplus W^{\prime} \rightarrow U_{1} \oplus S_{0} \oplus W^{\prime}$ is defined to be $U_{1} \oplus W \oplus W^{\prime}$ together with the composition

$$
\begin{array}{r}
V_{0} \oplus U_{1} \oplus S_{1} \oplus W \oplus W^{\prime} \stackrel{\theta \oplus \operatorname{id}_{S_{1} \oplus W^{\prime}}}{\longrightarrow} V_{1} \oplus U_{0} \oplus S_{1} \oplus W \oplus W^{\prime} \\
\stackrel{\phi \oplus \operatorname{id}_{V_{1}} \oplus W}{\longrightarrow} V_{1} \oplus U_{1} \oplus S_{0} \oplus W \oplus W^{\prime} .
\end{array}
$$

Clearly, we can add two stable vector bundles $\left(V_{0}, V_{1}\right) \oplus\left(W_{0}, W_{1}\right)=\left(V_{0} \oplus W_{0}, V_{1} \oplus\right.$ $W_{1}$ and $\left(V_{1}, V_{0}\right)$ is the additive inverse of $\left(V_{0}, V_{1}\right)$.

Let $W=\left(V_{0}, V_{1}\right)$ be a stable vector bundle of rank $d$ on the paracompact space $X$. There is a classifying map $c_{W}: X \rightarrow\{d\} \times B O$, which is constructed as follows. Let $\mu: B O \times B O \rightarrow B O$ be the Whitney sum map and $\iota: B O \rightarrow B O$ the inversion map. Let $c_{V_{0}}, c_{V_{1}}: X \rightarrow B O$ be two classifying maps. Then $c_{W}:=\mu \circ(\mathrm{id} \times \iota) \circ\left(c_{V_{0}}, c_{V_{1}}\right)$.

The Thom space of a vector bundle $V \rightarrow X$ is the space $\operatorname{Th}(V)=X^{V}=$ $\mathbb{D}(V) / \mathbb{S}(V)$, the quotient of the unit disc bundle by the unit sphere bundle. The Thom spectrum $\mathbb{T h}(W)$ of a stable vector bundle $W$ is produced as follows. Let $X_{n}:=c_{W}^{-1}\left(\{d\} \times B O_{d+n}\right)$; these subspaces form an exhaustive filtration $X_{-d} \subset$ $X_{1-d} \subset \cdots \subset X$. Let $W_{n}:=c_{W}^{*} \mathrm{£}_{d+n}$ be the pullback of the $d+n$-dimensional universal vector bundle. Clearly, there is an isomorphism $\left.W_{n+1}\right|_{X_{n}} \cong \mathbb{R} \oplus W_{n}$ and an isomorphism $W_{n} \oplus V_{1} \cong E_{0} \oplus \mathbb{R}^{n}$. The $n^{t h}$ space of $\mathbb{T h}(W)$ is the Thom space $X_{n}^{W_{n}}:=\mathbb{D}\left(W_{n}\right) / \mathbb{S}\left(W_{n}\right)$ of $W_{n}$ and the structure maps are

$$
\Sigma X_{n}^{W_{n}} \cong X_{n}^{\mathbb{R} \oplus W_{n}} \cong X_{n}^{\left.W_{n+1}\right|_{n}} \hookrightarrow X_{n+1}^{W_{n+1}} .
$$

The homotopy type of the spectrum $\mathbb{T h}(W)$ depends only on the homotopy class of $c_{W}$ alias isomorphism class of $W$. Furthermore, if $W \cong\left(W_{0}, 0\right)$, then the Thom spectrum is homotopy equivalent to the suspension spectrum $\Sigma^{\infty} X^{W_{0}}$ of the Thom space. In particular, the Thom spectrum of the trivial 0-dimensional bundle $\underline{0}$ on $X$ is $\Sigma^{\infty} X_{+}$. Moreover, if $W$ is an honest vector bundle and $V$ a stable vector bundle, then there is a map induced by the inclusion $\mathbb{T} \mathbf{h}(V) \rightarrow \mathbb{T} \mathbf{h}(V \oplus W)$.

2.2. The Madsen-Tillmann spectra. Let $n \geq 0$, let $B S O(n)$ be the classifying space for oriented Riemannian $n$-dimensional vector bundles and let $L_{n} \rightarrow B S O(n)$ be the universal oriented vector bundle. The reader should note that the space $B S O(0)$ is homotopy equivalent to the two-point space $\mathbb{S}^{0}$ and therefore it is not the classifying space for the group $S O(0)$. The most natural explanation for this phenomenon occurs in the framework of stacks. Let $\operatorname{Or}\left(\mathbb{R}^{n}\right)$ be the set of orientations of the vector space $\mathbb{R}^{n}$; the group $O(n)$ acts on $\operatorname{Or}\left(\mathbb{R}^{n}\right)$. The stack of oriented $n$ dimensional vector bundles is the quotient stack $\operatorname{Or}\left(\mathbb{R}^{n}\right) / / O(n)$. For $n \geq 1$, the $O(n)$-action on $\operatorname{Or}\left(\mathbb{R}^{n}\right)$ is transitive and hence $\operatorname{Or}\left(\mathbb{R}^{n}\right) / / O(n) \cong * / / S O(n)$, while for $n=0$, we have $\operatorname{Or}\left(\mathbb{R}^{0}\right) / / O(0) \cong \mathbb{S}^{0}$.

Definition 2.2.1. The Thom spectrum of the stable vector bundle $-L_{n}$ on $B O(n)$ is called the Madsen-Tillmann spectrum and it is denoted by $\operatorname{MTSO}(n)$. Moreover, we denote by $\operatorname{MNSO}(n)$ the Thom spectrum of $L_{n}$. 


\subsection{The Pontrjagin-Thom construction and the Madsen-Tillmann map.}

Let $M$ be a closed smooth oriented manifold of dimension $n$ and let $\operatorname{Diff}^{+}(M)$ be the group of diffeomorphisms of $M$ endowed with the Whitney $C^{\infty}$-topology. We will study smooth oriented $M$-bundles, i.e. fibre bundles $f: E \rightarrow B$ with structural group $\operatorname{Diff}^{+}(M)$ and fibre $M$. Let $Q \rightarrow B$ be the associated $\operatorname{Diff}(M)$ principal bundle. The vertical tangent bundle is the oriented vector bundle $T_{v} E:=$ $Q \times{ }_{\text {Diff }_{(M)}^{+}} T M \rightarrow Q \times \times_{\text {Diff }_{(M)}^{+}} M=E$. The normal bundle of $f$ is the stable vector bundle $\nu(f):=-T_{v} E$.

Let $\operatorname{Emb}^{f}\left(M ; \mathbb{R}^{\infty}\right)=\operatorname{colim}_{n} \operatorname{Emb}^{f}\left(M ; \mathbb{R}^{n}\right)$ be the space of fat embeddings of $M$ into $\mathbb{R}^{\infty}$ (a fat embedding is a pair, consisting of an embedding and a tubular neighborhood) endowed with the Whitney $C^{\infty}$-topology. It has a continuous $\operatorname{Diff}^{+}(M)$-action and by Whitney's embedding theorem and the tubular neighborhood theorem, $\operatorname{Emb}^{f}\left(M ; \mathbb{R}^{\infty}\right)$ is weakly contractible.

Therefore, provided $B$ is paracompact, the fibre bundle $Q \times_{\operatorname{Diff}^{+}(M)} \operatorname{Emb}^{f}\left(M ; \mathbb{R}^{\infty}\right) \rightarrow$ $B$ has a section and the space of sections is weakly contractible. Choose a section $j$; this is the same information as a fat embedding $j: E \rightarrow \mathbb{R}^{\infty} \times B$. For any $r \in \mathbb{N}$, let $B^{(r)}:=\left\{x \in B \mid j\left(f^{-1}(x)\right) \subset \mathbb{R}^{r}\right\}$; this is an exhaustion of $B$. Let $E^{(r)}:=f^{-1}\left(B^{(r)}\right)$. Let $U^{(r)} \subset \mathbb{R}^{r} \times B^{(r)}$ be the tubular neighborhood of the image of $j_{r}:=\left.j\right|_{E^{(r)}}$. The embedding $j_{r}$ induces a monomorphism $\left(j_{r}\right)_{*}: T_{v} E^{(r)} \rightarrow \mathbb{R}^{r} ; U$ is homeomorphic to the total space of $\mathbb{R}^{r} / T_{v} E^{(r)}$. Let $c: \mathbb{R}^{r} \times B^{(r)} \rightarrow U^{(r)} / \partial U^{(r)}$ the famous collapse map, which maps every point outside $U^{(r)}$ to the point represented by $\partial U^{(r)} ; c_{r}$ extends to a map $c_{r}: \Sigma^{r} B_{+}^{(r)} \rightarrow U^{(r)} / \partial U^{(r)}$. The target of $c_{r}$ is homeomorphic to the Thom space of $\mathbb{R}^{r} / T_{v} E^{(r)}$. As stable vector bundles on $E, \nu\left(j_{r}\right)$ and $\nu(f) \oplus \mathbb{R}^{r}$ are isomorphic. Therefore the maps $c_{r}$ assemble to a map of spectra

$$
\mathrm{PT}_{f}: \Sigma^{\infty} B_{+} \rightarrow \mathbb{T h}(\nu(f))
$$

the Pontrjagin-Thom map. The homotopy class of $\mathrm{PT}_{f}$ does not depend on the choice of the initial section $j$.

The original application of the Pontrjagin-Thom construction was to give a bordism theoretic description interpretation of the homotopy groups of Thom spectra (or vice versa). Let $V \rightarrow X$ be a stable vector bundle of dimension $n \in \mathbb{Z}$. Let $M$ be a closed oriented $n$-manifold, let $g: M \rightarrow X$ be map, let $\phi: T M \cong g^{*} V$ be an isomorphism of stable vector bundles and $-\phi: \nu_{M} \rightarrow-g^{*} V$ be induced isomorphism of the inverse bundles. Then the composition

$$
\Sigma^{\infty} \mathbb{S}^{0} \stackrel{\mathrm{PT}}{\rightarrow} \mathbb{T h}\left(\nu_{M}\right) \stackrel{\mathbb{T h}(-\phi)}{\rightarrow} \mathbb{T h}(-V)
$$

of the Pontrjagin-Thom map of the trivial bundle $M \rightarrow *$ with the map induced by $\phi$ defines a homotopy class in $\pi_{0}(\mathbb{T} \mathbf{h}(-V))$. Two such triples $\left(M_{i}, g_{i}, \phi_{i}\right), i=0,1$, define the same homotopy class provided that there is a bordism $N$ from $M_{0}$ to $M_{1}$, a map $h: N \rightarrow X$ with $\left.h\right|_{M_{i}}=g_{i}$ and a stable bundle isomorphism $\psi: T W \cong h^{*} V \oplus \mathbb{R}$ whose restriction to $M_{i}$ is the isomorphism $\left.T W\right|_{M_{i}} \cong T M_{i} \oplus \mathbb{R} \stackrel{g_{i} \oplus \text { id }_{\mathbb{R}}}{\cong} g_{i}^{*} V \oplus \mathbb{R}$ (the isomorphism $\left.T W\right|_{M_{0}} \cong T M_{0} \oplus \mathbb{R}$ identifies the inward normal vector with $1 \in \mathbb{R}$ and $\left.T W\right|_{M_{1}} \cong T M_{1} \oplus \mathbb{R}$ does the same to the outward normal vector). Converse, 
any homotopy class in $\pi_{0}\left(\mathbb{T} \mathbf{h}(-V)\right.$ arises in this fashion. Therefore, $\pi_{0}(\mathbb{T h}(-V))$ is the bordism group of such triples, with the bordism relation as above. Using the isomorphisms $\pi_{k}(\mathbb{T} \mathbf{h}(-V)) \cong \pi_{0}\left(\Sigma^{-k} \mathbb{T} \mathbf{h}(-V)\right)=\pi_{0}\left(\mathbb{T} \mathbf{h}\left(-\left(V \oplus \mathbb{R}^{k}\right)\right)\right)$, we get an interpretation of all homotopy groups. The details can be found in [35], ch. IV $\S 7$.

Now we return to a smooth oriented $M$-bundle $f: E \rightarrow B$. Recall that the space of orientation-preserving bundle maps $\lambda: T_{v} E \rightarrow L_{n}$ is contractible. Therefore the orientation defines a contractible space of maps $\mathbb{T h}(\lambda): \mathbb{T h}\left(-T_{v} E\right) \rightarrow \operatorname{MTSO}(n)$. The Madsen-Tilmann map of the bundle $f: E \rightarrow B$ is the composition

$$
\alpha_{E}:=\mathbb{T h}(\lambda) \circ \mathrm{PT}_{f}: \Sigma^{\infty} B_{+} \rightarrow \operatorname{MTSO}(n) .
$$

Under the adjunction between the functors $\Sigma^{\infty}$ and $\Omega^{\infty}, \alpha_{E}$ corresponds to a map $B \rightarrow \Omega^{\infty} \operatorname{MTSO}(n)$, which is the original Madsen-Tillmann map studied in [24], [25], [17]. We will call this adjoint by the same name and denote it by the same symbol. There is no danger of confusion, because we keep our notation for spaces and spectra entirely disjoint. However, we rarely use the infinite loop space point of view, which is so much emphasized by the papers [24, [25] and [17] (this is of course due to their main result). For the more computational purposes of the present paper, the spectra point of view is much more transparent and convenient.

For the universal oriented $M$-bundle $E_{M} \rightarrow B \operatorname{Diff}^{+}(M)$, we obtain a universal Madsen-Tillmann map

$$
\alpha_{E_{M}}: \Sigma^{\infty}\left(B \operatorname{Diff}^{+}(M)\right)_{+} \rightarrow \operatorname{MTSO}(n) .
$$

On the other extreme, the constant map $M \rightarrow *$ is a smooth oriented $M$-bundle and its Madsen-Tillmann map is a map $\alpha_{M}: \Sigma^{\infty} \mathbb{S}^{0} \rightarrow \operatorname{MTSO}(n)$.

2.4. Some homotopy properties of Madsen-Tillmann spectra. There are several maps which relate the spectra $\operatorname{MTSO}(n)$ for different values of $n$.

The obvious bundle isomorphism $\left.L_{n}\right|_{B S O(n-1)} \cong L_{n-1} \oplus \mathbb{R}$ induces a map of spectra $\eta: \operatorname{MTSO}(n-1) \rightarrow \Sigma \operatorname{MTSO}(n)$.

Furthermore, the inclusion $-L_{n} \rightarrow \underline{0}$ of stable vector bundles on $B S O(n)$ yields a spectrum map $\omega: \operatorname{MTSO}(n) \rightarrow \Sigma^{\infty} B S O(n)_{+}$. If $f: E \rightarrow B$ is an oriented manifold bundle, then the composition $\Sigma^{\infty} B_{+} \stackrel{\alpha_{E}}{\rightarrow} \operatorname{MTSO}(n) \stackrel{\omega}{\rightarrow} \Sigma^{\infty} \mathrm{BSO}(n)_{+}$is the composition of the Becker-Gottlieb transfer $\Sigma^{\infty} B_{+} \rightarrow \Sigma^{\infty} E_{+}$(see [13]) with the classifying map $\Sigma^{\infty} E_{+} \rightarrow \Sigma^{\infty} \mathrm{BSO}(n)_{+}$of $T_{v} E$. Therefore, if $B$ is connected (and $n>0$ ), then the induced map on $\mathbb{Z}=\pi_{0} \Sigma^{\infty} B_{+} \rightarrow \Sigma^{\infty} B S O(n)_{+}=\mathbb{Z}$ is the multiplication by the Euler number $\chi(M)$ of $M$, by Theorem 2.4 of [13].

The Madsen-Tillmann map of the oriented $\mathbb{S}^{n-1}$-bundle $B S O(n-1) \rightarrow B S O(n)$ is a map $\beta: \Sigma^{\infty} B S O(n)_{+} \rightarrow \operatorname{MTSO}(n-1)$.

Proposition 2.4.1. The maps $\eta, \omega$ and $\beta$ form a cofibration sequence

$$
\operatorname{MTSO}(n) \stackrel{\omega}{\rightarrow} \Sigma^{\infty} B S O(n)_{+} \stackrel{\beta}{\rightarrow} \operatorname{MTSO}(n-1) \stackrel{\eta}{\rightarrow} \Sigma \operatorname{MTSO}(n)
$$


Proof. This follows immediately from Lemma 2.1 in [16].

The (homotopy) colimit of the sequence

$$
\operatorname{MTSO}(0) \stackrel{\eta}{\rightarrow} \Sigma \operatorname{MTSO}(1) \stackrel{\eta}{\rightarrow} \Sigma^{2} \operatorname{MTSO}(2) \rightarrow \ldots
$$

is the universal Thom spectrum $\widetilde{\mathrm{MSO}}$, the Thom spectrum of the universal 0dimensional stable vector bundle $-L \rightarrow$ BSO (which becomes $\mathbb{R}^{n}-L_{n}$ when restricted to $\mathrm{BSO}(n))$. The usual universal Thom spectrum MSO is the Thom spectrum of $L \rightarrow$ BSO. The spectra $\widetilde{\mathrm{MSO}}$ and MSO are homotopy equivalent: Let $\iota: \mathrm{BSO} \rightarrow \mathrm{BSO}$ be the inversion map, such that $\iota^{*} L=-L$. The map $\iota$ is covered by a bundle map $j:-L \rightarrow L$ which induces a homotopy equivalence $\mathrm{Th}(j): \widehat{\mathrm{MSO}} \rightarrow \mathrm{MSO}$

The long exact homotopy sequence induced by 2.4 .2 shows that the map $\eta_{*}$ : $\pi_{i}(\operatorname{MTSO}(n-1)) \rightarrow \pi_{i}\left(\sum \operatorname{MTSO}(n)\right)$ is an epimorphism if $i \leq 0$ and an isomorphism if $i<0$. Therefore the inclusion $\Sigma^{n} \operatorname{MTSO}(n) \rightarrow \widetilde{\mathrm{MSO}}$ yields an isomorphism $\pi_{i}(\mathrm{MTSO}(n)) \cong \pi_{n+i}(\widetilde{\mathrm{MSO}}) \cong \pi_{n+i}(\mathrm{MSO}) \cong \Omega_{n+i}^{S O}$ (the oriented bordism group) for all $i<0$. Therefore from 2.4.1, we get an exact sequence

$$
\pi_{0}(\operatorname{MTSO}(n)) \rightarrow \pi_{0}\left(\Sigma^{\infty} \operatorname{BSO}(n)_{+}\right) \rightarrow \pi_{0}(\operatorname{MTSO}(n-1)) \rightarrow \pi_{-1}(\operatorname{MTSO}(n)) \rightarrow 0
$$

or

$$
\pi_{0}(\operatorname{MTSO}(n+1)) \rightarrow \mathbb{Z} \rightarrow \pi_{0}(\operatorname{MTSO}(n)) \rightarrow \Omega_{n}^{S O} \rightarrow 0
$$

In order to study these groups further, we use the bordism-theoretic interpretation of $\pi_{0}(\operatorname{MTSO}(n))$ from section 2.3. We can rephrase it in such a way that $\pi_{0}(\operatorname{MTSO}(n))$ is the bordism group of oriented $n$-manifolds, where $M_{0}$ and $M_{1}$ are considered to be bordant if and only if there exists an oriented bordism $N$ between them, an oriented $n$-dimensional vector bundle $V$ on $N$ and a stable bundle isomorphism $T N \cong V \oplus \mathbb{R}$. Clearly we can assume that $N$ has no closed component and therefore, there is an actual isomorphism of vector bundles $T N \cong V \oplus \mathbb{R}$. In other words, there is a nowhere vanishing tangential vector field on $N$ which is the inward normal vector field on $M_{0}$ and the outward normal vector field on $M_{1}$. This bordism group is also known as Reinhardt's bordism group, see [34]. We remark that, given an oriented $n$-manifold $M$, the homotopy class of the Madsen-Tillmann map $\Sigma^{\infty} \mathbb{S}^{0} \rightarrow \operatorname{MTSO}(n)$ of the trivial bundle $M \rightarrow *$ coincides with the bordism class of $M$ under the above isomorphism.

The maps in 2.4 .3 have the following interpretation:

(1) $\pi_{0}(\operatorname{MTSO}(n+1)) \rightarrow \mathbb{Z}$ sends the bordism class of an oriented $n+1$-manifold $M$ to its Euler number $\chi(M)$.

(2) $\mathbb{Z} \rightarrow \pi_{0}(\operatorname{MTSO}(n))$ sends 1 to the bordism class of $\mathbb{S}^{n}$.

(3) $\pi_{0}(\operatorname{MTSO}(n)) \rightarrow \Omega_{n}^{S O}$ is the forgetful map. 
Now let $\operatorname{Eul}_{n} \subset \mathbb{Z}$ be the subgroup generated by all Euler numbers of oriented $n$-manifolds; the exact sequence 2.4 .3 induces

$$
0 \rightarrow \mathbb{Z} / \operatorname{Eul}_{n+1} \rightarrow \pi_{0}(\operatorname{MTSO}(n)) \rightarrow \Omega_{n}^{S O} \rightarrow 0 .
$$

The group $\operatorname{Eul}_{n} \subset \mathbb{Z}$ is easily computed. Its values are

$$
\operatorname{Eul}_{n}=\left\{\begin{array}{lll}
0 ; & n \neq 0 & (\bmod 2) \\
2 \mathbb{Z} ; & n \equiv 2 & (\bmod 4) \\
\mathbb{Z} ; & n \equiv 0 & (\bmod 4)
\end{array}\right.
$$

The first case is clear. The third case follows from $\chi\left(\mathbb{S}^{4 k}\right)=2$ and $\chi\left(\mathbb{C P}^{2 k}\right)=$ $2 k+1$. The second case is implied by $\chi\left(\mathbb{S}^{4 k+2}\right)=2$ and the congruence $\chi\left(M^{4 k+2}\right) \equiv 0$ (mod 2) which follows from Poincaré duality in a straightforward manner. Now we conclude:

(1) If $n \equiv 3(\bmod 4)$, then $\pi_{0}(\operatorname{MTSO}(n)) \cong \Omega_{n}^{S O}$.

(2) If $n \equiv 2(\bmod 4)$, then the sequence 2.4 .4 splits by $\pi_{0}(\operatorname{MTSO}(n)) \rightarrow \mathbb{Z}$; $[M] \mapsto \frac{1}{2} \chi(M)$.

(3) If $n \equiv 0(\bmod 4)$, then 2.4 .4 is also split. If $M^{4 m}$ is an oriented manifold, then $\operatorname{sign}(M)+\chi(M) \equiv 0(\bmod 2)$ is immediate from the definition of the signature and the Euler number and from Poincaré duality. The map $\pi_{0}(\operatorname{MTSO}(n)) \rightarrow \mathbb{Z},[M] \mapsto \frac{1}{2}(\operatorname{sign}(M)+\chi(M))$ is a splitting.

(4) If $n \equiv 1(\bmod 4)$, then 2.4 .4 is split by the Kervaire semi-characteristic Kerv $: \pi_{0}(\operatorname{MTSO}(n)) \rightarrow \mathbb{Z} / 2$. For a $(4 m+1)$-manifold, the Kervaire semicharacteristic is $\operatorname{Kerv}(M):=\sum_{i>0} \operatorname{dim} H_{2 i}(M ; \mathbb{R})=\sum_{i=0}^{2 m} \operatorname{dim} H_{i}(M ; \mathbb{R})$ $(\bmod 2)$

Proposition 2.4.5. The Kervaire semi-characteristic of an oriented $4 m+1$-manifold $M$ only depends on its bordism class in $\pi_{0}(\operatorname{MTSO}(4 m+1))$.

Proof. It is enough to show the following: If $N^{4 m+2}$ is a connected oriented manifold with boundary $M$ and if there is a nowhere vanishing vector field on $N$ which is normal to the boundary, then $\operatorname{Kerv}(M)=0$. Clearly, the double $d N$ of $N$ is closed and has a vector field without zeroes; thus $\chi(d N)=0$ and therefore $\chi(N)=0$. Now look at the long exact sequence of the pair $(N, M)$ in real cohomology:

$0 \rightarrow H^{0}(N, M) \rightarrow H^{0}(N) \rightarrow H^{0}(M) \rightarrow \ldots \rightarrow H^{2 m}(M) \rightarrow H^{2 m+1}(N, M) \rightarrow A \rightarrow 0$,

where $A$ is the image of $H^{2 m+1}(M, N) \rightarrow H^{2 m+1}(N)$. By Poincaré duality, $\operatorname{dim} H^{i}(N, M ; \mathbb{R})=\operatorname{dim} H^{4 m+2-i}(N ; \mathbb{R})$. Therefore, by exactness of the preceding sequence:

$$
\begin{aligned}
\operatorname{Kerv}(M)+\operatorname{dim} A & =\chi(N)+\operatorname{Kerv}(M)+\operatorname{dim} A \equiv \\
\sum_{i=0}^{4 m+2} \operatorname{dim} H^{i}(N, \mathbb{R})+\sum_{i=0}^{2 m} \operatorname{dim} H^{i}(M ; \mathbb{R})+\operatorname{dim} A & \equiv \quad 0 \quad(\bmod 2) .
\end{aligned}
$$


But the cup-product pairing on $A$ is skew-symmetric and nondegenerate by Poincaré duality; thus $\operatorname{dim} A$ is even.

2.5. Thom isomorphisms. Let $V \rightarrow X ; W \rightarrow Y$ be two stable vector bundles. There is a canonical homotopy equivalence $\mathbb{T h}(V) \wedge \mathbb{T} \mathbf{h}(W) \simeq \mathbb{T h}(V \times W)$. If $X=Y$, we get a diagonal map $\operatorname{diag}: \mathbb{T} \mathbf{h}(V \oplus W) \rightarrow \mathbb{T} \mathbf{h}(V) \wedge \mathbb{T} \mathbf{h}(W)$. A special case is the diagonal $\mathbb{T h}(V) \rightarrow \Sigma^{\infty} X_{+} \wedge \mathbb{T} \mathbf{h}(V)$.

Now assume that $A$ is an associative and commutative ring spectrum with unit (the rather old-fashioned notion of [3] is sufficient for our purposes). Let $V \rightarrow X$ be a stable vector bundle of rank $d \in \mathbb{Z}$. The cohomology $A^{*}(\mathbb{T} \mathbf{h}(V))$ is a graded right $A^{*}(X)$-module; a pair $(x, y) \in A^{n}(X) \times A^{m}(\mathbb{T} \mathbf{h}(V)$ is sent to the composition

$$
x \cdot y: \mathbb{T h}(V) \stackrel{\operatorname{diag}}{\rightarrow} \Sigma^{\infty} X_{+} \wedge \mathbb{T} \mathbf{h}(V) \stackrel{x \wedge y}{\rightarrow} \Sigma^{n} A \wedge \Sigma^{m} A \rightarrow \Sigma^{n+m} A .
$$

A Thom class of $V$ with $A$-coefficients is a cohomology class $v \in A^{d}(\mathbb{T h}(V))$ such that for any $x \in X$, the image of $v$ under the restriction map $A^{d}(\mathbb{T h}(V)) \rightarrow$ $A^{d}\left(\mathbb{T} \mathbf{h}\left(V_{x}\right)\right) \cong A^{d}\left(\mathbb{S}^{d}\right) \cong A^{0}(*)$ is a unit. This is equivalent to saying that $A^{*}(\mathbb{T} \mathbf{h}(V))$ is a free $A^{*}(X)$-module on the generator $v$. The isomorphism $\operatorname{th}_{V}^{A}: A^{*}(X) \rightarrow$ $A^{*+d}(\mathbb{T h}(V)) ; x \mapsto x \cdot v$ is called the Thom isomorphism. If $A$ is understood, then the superscript is often omitted.

More generally, we can define a relative Thom isomorphism. Let $V$ be a stable vector bundle of rank $d$ and let $W$ be another stable vector bundle of rank $e$. Assume that $V$ has a Thom class $v$. Let $\operatorname{th}_{W, W \oplus V}^{A}: A^{*}(\mathbb{T h}(W)) \rightarrow A^{*+d}(\mathbb{T h}(W \oplus V))$ be the homomorphism which maps $x \in A^{n}(\mathbb{T} \mathbf{h}(W))$ to the composition

$$
\mathbb{T} \mathbf{h}(W \oplus V) \stackrel{\operatorname{diag}}{\rightarrow} \mathbb{T h}(W) \wedge \mathbb{T h}(V) \stackrel{x \wedge v}{\rightarrow} \Sigma^{n} A \wedge \Sigma^{d} A \rightarrow \Sigma^{n+d} A ;
$$

this is an isomorphism of $A^{*}(X)$-modules. If $v \in A^{d}(\mathbb{T h}(V))$ and $w \in A^{e}(\mathbb{T h}(W))$ are Thom classes, then the composition

$$
\mathbb{T h}(V \oplus W) \stackrel{\operatorname{diag}}{\rightarrow} \mathbb{T h}(V) \wedge \mathbb{T h}(W) \stackrel{v \wedge w}{\rightarrow} \Sigma^{d} A \wedge \Sigma^{e} A \rightarrow \Sigma^{d+e} A
$$

represents a Thom class for $V \oplus W$. If the Thom classes are chosen compatibly in this way, then the Thom isomorphisms are compatible in the sense that $\operatorname{th}_{U \oplus V, U \oplus V \oplus W} \circ \operatorname{th}_{U, U \oplus V}=\operatorname{th}_{U, U \oplus V \oplus W}$ whenever $U$ is an arbitrary stable vector bundle. We shall use the short notation $\operatorname{th}_{V}: A^{*}(\mathbb{T h}(W)) \rightarrow A^{*}(\mathbb{T h}(V \oplus W)$ if $W$ is understood.

Examples: The examples of ring spectra which play a role in this paper are Eilenberg-Mac Lane spectra $H R$ for commutative rings $R$ as well as the complex $K$-theory spectrum $K$. It is well-known that a vector bundle which is oriented in the ordinary sense has a preferred $H \mathbb{Z}$-Thom class and so it has a $H R$-Thom class for any ring $R$. A stable vector bundle has an $H \mathbb{Z}$-orientation if and only if $w_{1}(V)=0$. Any complex vector bundle has a $K$-orientation and so does every complex stable vector bundle, i.e. a formal difference of complex vector bundles. However, there are several choices for these $K$-orientation. We follow the convention that the Thom class of a complex vector bundle $\pi: V \rightarrow X$ of rank $n$ is represented by the complex 


$$
0 \rightarrow \pi^{*} \Lambda^{0} V \stackrel{v \wedge}{\rightarrow} \pi^{*} \Lambda^{1} V \stackrel{v \wedge}{\rightarrow} \pi^{*} \Lambda^{2} V \rightarrow \ldots \pi^{*} \Lambda^{n} V \rightarrow 0 .
$$

The umkehr map: Let $f: E \rightarrow B$ be a smooth fibre bundle of dimension $n$ and we assume that $\nu(f)$ has an $A$-orientation. Then we can define the umkehr map in $A$-cohomology. Let $f_{!}: A^{*}(E) \rightarrow A^{*-n}(B)$ be the composition

$$
A^{*}(E) \stackrel{\mathrm{th}_{\nu(f)}}{\rightarrow} A^{*-n}(\mathbb{T h}(\nu(f))) \stackrel{\mathrm{PT}_{f}^{*}}{\rightarrow} A^{*-n}(B)
$$

2.6. Cohomology of Madsen-Tillmann spectra. There is the Thom isomorphism in ordinary cohomology:

$$
H^{*}(B S O(n) ; R)=H^{*}\left(\Sigma^{\infty} B S O(n)_{+} ; R\right) \cong H^{*-n}(\operatorname{MTSO}(n) ; R) .
$$

The cohomology of $B S O(n)$ is well known, at least if $R=\mathbb{F}_{2}$ or $R=\mathbb{Q}$. In the first case

$$
H^{*}\left(B S O(n) ; \mathbb{F}_{2}\right) \cong \mathbb{F}_{2}\left[w_{2}, w_{3}, \ldots w_{n}\right]
$$

and in the second case, we have

$$
H^{*}(B S O(2 m+1) ; \mathbb{Q}) \cong \mathbb{Q}\left[p_{1}, p_{2}, \ldots p_{m}\right]
$$

and

$$
H^{*}(B S O(2 m) ; \mathbb{Q}) \cong \mathbb{Q}\left[p_{1}, \ldots p_{m}, \chi\right] /\left(\chi^{2}-p_{m}\right) .
$$

Let $f: E \rightarrow B$ be an oriented $n$-dimensional manifold bundle, let $\alpha_{E}: \Sigma^{\infty} B_{+} \rightarrow$ $\operatorname{MTSO}(n)$ be its Madsen-Tillmann map. An element $c \in H^{*}(B S O(n) ; R)$ can be interpreted as a characteristic class for oriented $n$-dimensional vector bundles and therefore we write $c\left(T_{v} E\right) \in H^{*}(E)$ for the pullback $\bar{\lambda}^{*} c$, where $\bar{\lambda}: E \rightarrow B S O(n)$ is any map underlying a bundle map $T_{v} E \rightarrow L_{n}$.

Proposition 2.6.1. Let the notations be as above. Then

$$
\alpha_{E}^{*} \operatorname{th}_{-L_{n}}(c)=f_{!}\left(c\left(T_{v}(E)\right)\right) \in H^{*-n}(B) .
$$

Proof. By definition

$$
\alpha^{*} \operatorname{th}_{-L_{n}}(c) \stackrel{2.3 .1}{=} \mathrm{PT}_{f}^{*} \mathbb{T h}(\lambda)^{*} \operatorname{th}_{-L_{n}}(c)=\mathrm{PT}_{f}^{*} \operatorname{th}_{-T_{v} E}\left(c\left(T_{v} E\right)\right) .
$$

The second equality expresses the compatibility of Thom isomorphisms and pullbacks. 
Therefore any $c \in H^{*}(B S O(n))$ defines a characteristic class of oriented $n$ manifold bundles. We call these classes "generalized MMM classes", because the case $n=2, c=\chi^{i+1}$ gives the classes $\kappa_{i}$ defined by $M$ umford [31], Miller [29] and $M$ orita [30].

The bundle $L_{n}$ is not $K$-orientable, but nevertheless there is the relative Thom isomorphism

$K^{*}(\operatorname{MTSO}(n))=K^{*}\left(\mathbb{T h}\left(-L_{n}\right)\right) \cong K^{*}\left(\mathbb{T h}\left(-L_{n} \oplus L_{n} \otimes \mathbb{C}\right)=K^{*}\left(\mathbb{T h}\left(L_{n}\right)\right)=K^{*}(\operatorname{MNSO}(n))\right.$,

which plays an essential role in the following sections. Note that we used Bott periodicity to identify $K^{*}$ with $K^{*+2}$. We will do this throughout the paper.

2.7. Infinite loop spaces. Recall the adjunction between the two functors $\Sigma^{\infty}$ and $\Omega^{\infty}$ : given a spectrum $\mathbf{E}$ and a space $X$, there is a natural bijection

$$
\left[X, \Omega^{\infty} \mathbf{E}\right] \cong\left[\Sigma^{\infty} X_{+}, \mathbf{E}\right]
$$

The adjoint $\Sigma^{\infty}\left(\Omega^{\infty} \mathbf{E}\right)_{+} \rightarrow \mathbf{E}$ of the identity on $\Omega^{\infty} \mathbf{E}$ induces a map

$$
s: A^{*}(\mathbf{E}) \rightarrow A^{*}\left(\Omega^{\infty} \mathbf{E}\right),
$$

the cohomology suspension, whenever $A$ is a multiplicative commutative ring spectrum. The left-hand-side is only a graded $A^{*}$-module, but the right-hand-side is a graded-commutative $A^{*}$-algebra (if $A$ is multiplicative and commutative). Let $\Lambda$ denote the functor which associates to a graded module the free, graded-commutative algebra it generates; $s$ extends to an algebra homomorphism

$$
s: \Lambda\left(A^{*>0}(\mathbf{E} ; R)\right) \rightarrow A^{*}\left(\Omega_{0}^{\infty} \mathbf{E}: R\right)
$$

If $A=H \mathbb{Q}$, the extended $s$ is an isomorphism by a classical result of algebraic topology, see [27], p. $262 \mathrm{f}$.

If $X$ is a space and $\Sigma^{\infty} X_{+} \rightarrow \mathbf{E}$ a map with adjoint $X \rightarrow \Omega^{\infty} \mathbf{E}$, then the following diagram commutes

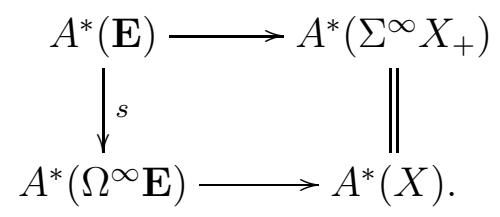




\section{BACKGROUND MATERIAL ON INDEX THEORY}

In this section, we will present background material on index theory for bundles of compact manifolds. For details, the reader is referred to either the original source [10] or to the textbook [23].

There are two types of the $K$-theoretic index theorem: One for usual elliptic operators and another one for self-adjoint elliptic operators on a fibre bundle $f$ : $E \rightarrow B$. In the former case, the index is an element in $K^{0}(B)$ while in the latter one we get an index in $K^{1}(B)$.

Assume that $f: E \rightarrow B$ is a smooth fibre bundle on a compact space $B$ (the assumptions on $B$ can be weakened) with compact closed fibres. Assume that a fibrewise smooth Riemannian metric on the vertical tangent bundle $T_{v} E$ is chosen. All vector bundles on $E$ will be fibrewise smooth (i.e. the transition functions are smooth in the fibre-direction) and all hermitian metrics on vector bundles are understood to be smooth. All differential operations, like exterior derivatives and connections, will be fibrewise.

For an hermitian vector bundle $V \rightarrow E$, we denote $\Gamma_{B}(V)=\bigcup_{x \in B} \Gamma\left(E_{x} ; V_{x}\right)$, where $E_{x}=f^{-1}(x)$ and $V_{x}=\left.V\right|_{E_{x}}$. This family of vector spaces over $B$ can be made into a vector bundle (of Fréchet spaces) by requiring that a section $s: B \rightarrow \Gamma_{B}(V)$ is continuous if the associated section of $V \rightarrow E$ is continuous in the $C^{\infty}$-topology. Using the metrics on $T_{v} E$ and $V$ and a connection on $V$, we can define the $L^{2}$ Sobolev norms $\|\ldots\|_{r}$ on $\Gamma_{B}(V)$, for all $r \geq 0$. The completion with respect to this norms is a Hilbert bundle which we denote by $W_{B}^{2, r}(V)$.

There is a technical problem to overcome at this point; it is discussed and solved in [7, pp. 5, 13 f., 38-43. Namely, it is not quite true that the structural group of $W_{B}^{2, r}(V)$ is general linear group of an infinite-dimensional Hilbert space. The reason is that the action $\operatorname{Diff}(M) \curvearrowright W^{2, s}(M)$ is continuous only in the sense that $\operatorname{Diff}(M) \times$ $W^{2, s}(M) \rightarrow W^{2, s}(M)$ is continuous, but not $\operatorname{Diff}(M) \rightarrow \mathrm{GL}\left(W^{2, s}\right)$ when the latter has the norm topology. Instead, this map is continuous when $\operatorname{GL}\left(W^{2, s}\right)$ has the compactly generated compact-open topology. Denote by $\operatorname{GL}\left(W^{2, s}\right)_{c o}$ the group with this topology. Then $\mathrm{GL}\left(W^{2, s}\right)_{c o}$ is contractible (this is much easier than Kuiper's theorem which asserts that $\mathrm{GL}\left(W^{2, s}\right)$ is contractible). Moreover, $\operatorname{GL}\left(W^{2, s}\right)_{c o}$ acts continuously by conjugation on the space of Fredholm operators with a suitably redefined topology. This new space of Fredholm operators is homotopy equivalent to the original one.

Therefore the Hilbert bundles $W_{B}^{2, r}(V)$ are trivial and the trivialization is unique up to homotopy (in fact, the space of trivializations is contractible).

Let $V_{0}, V_{1} \rightarrow E$ be two hermitian vector bundles and let $D: V_{0} \rightarrow V_{1}$ be a vertical elliptic operator of order $m$. Then $D$ has an extension to the bundle of Sobolev spaces: $D: W_{B}^{2, s+m}(V) \rightarrow W_{B}^{2, s}(V)$, which consist of Fredholm operators. Now we choose, for any vector bundle $V$, an elliptic pseudodifferential operator $A$ of order $-m / 2$ which is invertible (for example $A_{V}=\left(1+\nabla^{*} \nabla\right)^{-m / 4}$ will do for any connection $\nabla$ on $V$ ). Now the operator $A_{V_{1}} D A_{V_{0}}$ has order 0 and so it induces

\footnotetext{
${ }^{1}$ We are using the Bott periodicity theorem without mentioning it. Therefore we identify $K^{1}$ with $K^{-1}$.
} 
a family of Fredholm operator $W_{B}^{2,0}\left(V_{0}\right) \rightarrow W_{B}^{2,0}\left(V_{1}\right)$. After an application of the trivializations above, we get a continuous map, denoted ind $(D)$ :

$$
\operatorname{ind}(D): B \rightarrow \operatorname{Fred}(H),
$$

where $H$ is a fixed separable, infinite-dimensional Hilbert space. The AtiyahJänich theorem states that the $\operatorname{Fred}(H)$ is a classifying space for complex $K$-theory and therefore we get an element ind $(D) \in K^{0}(B)$. It does not depend on the choices involves.

On the other hand, if $D: \Gamma_{B}(V) \rightarrow \Gamma_{B}(V)$ is a formally self-adjoint elliptic operator of order $m \geq 0$, we get an index in $K^{1}(B)$. Here we consider the operator $A_{V} D A_{V}^{*}$, which is elliptic of order 0 and formally self-adjoint. It has the same kernel and the same positive and negative spectral spaces as the original $D$.

Thus we get a self-adjoint bounded Fredholm operator $D: W_{B}^{2,0}(V) \rightarrow W_{B}^{2,0}(V)$. In the same way as for ordinary elliptic operators, we get a map $B \rightarrow \operatorname{Fred}_{s . a}(H)$, where $\operatorname{Fred}_{\text {s.a. }}(H)$ is the space of self-adjoint Fredholm operators on $H$ endowed with the norm topology. Let $\operatorname{Fred}_{s . a .}^{ \pm}(H) \subset \operatorname{Fred}_{s . a .}(H)$ be the subspace consisting operators $A$ such that $\pm A$ is essentially positive (an operator is essentially positive if there exists an $A$-invariant subspace $U \subset H$ of finite codimension, such that $\left.A\right|_{U}$ is positive definite). The two spaces $\operatorname{Fred}_{s . a}^{ \pm}(H) \subset \operatorname{Fred}_{\text {s.a. }}(H)$ are open, closed and contractible (in fact, $\operatorname{Fred}_{s . a .}^{ \pm}(H)$ is star-shaped with center $\pm \mathrm{id}$ ).

Let $\operatorname{Fred}_{\text {s.a. }}^{0}=\operatorname{Fred}_{\text {s.a. }}(H) \backslash\left(\operatorname{Fred}_{\text {s.a. }}^{+}(H) \cup \operatorname{Fred}_{\text {s.a. }}^{-}(H)\right)$. Atiyah and Singer [12] showed that it has a very interesting topology: it has the homotopy type of the infinite unitary group $U(\infty)$. Thus it is a representing space for $K^{-1}$.

Returning to the self-adjoint family of operators $D$ on $E \rightarrow B$, the map $B \rightarrow$ $\operatorname{Fred}_{\text {s.a. }}(H)$ defines an element $\operatorname{ind}(D) \in K^{1}(B)$ (if $D$ is essentially definite, this element is trivial).

3.1. The topological index. Let $f: E \rightarrow B$ be a smooth proper bundle, $\pi$ : $T:=T_{v}^{*} E \rightarrow E$ the vertical cotangent bundle and $\pi_{0}: \mathbb{S}\left(T_{v}^{*} E\right) \rightarrow E$ its unit sphere bundle. Let $D: \Gamma_{B}\left(V_{0}\right) \rightarrow \Gamma_{B}\left(V_{1}\right)$ an elliptic differential operator. Recall that the symbol of $D$ is a bundle map $\operatorname{smb}_{D}: \pi^{*} V_{0} \rightarrow \pi^{*} V_{1}$ which is an isomorphism outside the zero section (this is the definition of ellipticity). If $D$ has order 1 , then the symbol is

$$
\operatorname{smb}_{D}(\xi) v=i(D(f s)-f D s)
$$

where $\xi$ is a vertical cotangent vector at $x \in E, f$ is a smooth function such that $d f_{x}=\xi$ and $s$ is a section of $V_{0}$ such that $s(x)=v$. For higher orders, there is a more complicated formula, which we will not need here.

We will constantly identify the vertical cotangent and the vertical tangent bundle. The symbol $\operatorname{smb}_{D}$ defines the symbol class $\left[\operatorname{smb}_{D}\right]_{0} \in K^{0}(T ; T \backslash 0)=K^{0}\left(E^{T}\right)$ of $D$.

Following [6], we can associate a symbol class $\left[\operatorname{smb}_{D}\right]_{1} \in K^{-1}\left(E^{T}\right)$ to a self-adjoint elliptic operator $D$. Consider the symbol $\operatorname{smb}_{D}: \pi^{*} V \rightarrow \pi^{*} V$. It is a self-adjoint endomorphism of $\pi^{*} V$ and it is an isomorphism away from the zero section. Let $\tilde{\pi}$ : $T \oplus \mathbb{R} \rightarrow E$. We define $\left[\mathrm{smb}_{D}\right]_{1}$ to be the class in $K^{-1}\left(E^{T}\right)=K^{0}((T, T \backslash 0) \times(\mathbb{R}, \mathbb{R} \backslash 0))$ represented by the complex 


$$
0 \rightarrow \tilde{\pi}^{*} V \stackrel{\mathrm{smi}_{D}}{\rightarrow} \tilde{\pi}^{*} V \rightarrow 0
$$

where $\operatorname{smb}_{D}$ is given at the point $(x, t) \in T \oplus \mathbb{R}$ by $\operatorname{smb}_{D(x, t)}:=\left(\operatorname{smb}_{D}\right)_{x}-i t \mathbf{1}$. Actually, [6] give a different formula, but the passage between the two formulations is by an elementary deformation. We leave it to the reader to figure that out.

The bundle $T_{v} E$ is not necessarily $K$-orientable, but the bundle $T_{v} E \oplus T_{v} E \cong$ $T_{v} E \otimes \mathbb{C}$ is $K$-oriented. Therefore there is the Thom isomorphism th- $T_{v} E \otimes \mathbb{C}:$ $K^{*}\left(E^{T_{v} E}\right) \rightarrow K^{*-2 d}\left(E^{-T_{v} E}\right)$.

The Atiyah-Singer family index theorem ([10] for the usual case, [6] for the selfadjoint case) states that

$$
\operatorname{ind}(D)=\beta^{-d} \mathrm{PT}_{f}^{*} \text { th }_{-T_{v} E \otimes \mathbb{C}}\left(\left[\operatorname{smb}_{D}\right]_{i}\right) \in K^{i}(B)
$$

in both cases $(i=0,1)$, where $\beta: K^{0} \rightarrow K^{-2}$ denotes the Bott periodicity isomorphism.

3.2. Universal operators. Now we assume that the first order elliptic operator $D$ on the $n$-dimensional oriented bundle $E \rightarrow B$ family is universal on the symbolic level. By that expression, we mean that there exist $S O(n)$-representations $W_{0}$ and $W_{1}$ and an $S O(n-1)$-equivariant map $\gamma: W_{0} \rightarrow W_{1}$ such that

(1) as Hermitian vector bundles, $V_{0}$ and $V_{1}$ are isomorphic to the associated bundle $\operatorname{Fr}_{v}(E) \times_{S O(n)} W_{i} \rightarrow E$;

(2) the symbol $\mathrm{smb}_{D}$ restricted to the unit cotangent sphere bundle equals the bundle map $\operatorname{Fr}_{v}(E) \times_{S O(n-1)} W_{0} \rightarrow \operatorname{Fr}_{v}(E) \times_{S O(n-1)} W_{1}$ induced by $\gamma$.

The trivial vector bundles $V_{i} \times \mathbb{R}^{n}$ on $\mathbb{R}^{n}$ are $S O(n)$-equivariant and the map $\gamma$ defines an $S O(n)$-equivariant isomorphism $V_{0} \times \mathbb{S}^{n-1} \rightarrow V_{1} \times \mathbb{S}^{n-1}$. Therefore, $\left(V_{0}, V_{1}, \gamma\right)$ defines a class $\sigma_{D} \in K_{S O(n)}^{0}\left(\mathbb{D}^{n}, \mathbb{S}^{n-1}\right)$. The image of $\sigma_{D}$ under the standard homomorphism $K_{S O(n)}^{0}\left(\mathbb{D}^{n}, \mathbb{S}^{n-1}\right) \rightarrow K^{0}\left(\mathbb{D}\left(L_{n}\right), \mathbb{S}\left(L_{n}\right)\right) \cong K^{0}(\operatorname{MNSO}(n))$ is denoted by the same symbol. By the Thom isomorphism $K^{0}(\operatorname{MNSO}(n)) \cong K^{0}(\operatorname{MTSO}(n))$, we get a class th $\sigma_{D} \in K^{0}(\operatorname{MTSO}(n))$.

The index theorem for the symbolically universal operator $D$ on the fibre bundle $f: E \rightarrow B$ now reads:

$$
\operatorname{ind}(D)=\alpha_{E}^{*} \text { th } \sigma_{D}
$$

Similarly, if $V_{0}=V_{1}=V$ and $\gamma$ is self-adjoint, we get a class $\sigma_{D} \in K^{1}(\operatorname{MNSO}(n))$ and th $\sigma \in K^{1}(\operatorname{MTSO}(n))$ and the index theorem is as in 3.2.1. 


\section{THE INDEX THEOREM FOR THE ODD SIGNATURE OPERATOR}

4.1. The signature operators. Let $M$ be a closed oriented Riemannian manifold of dimension $n$. Recall that there is the Hodge star operator $\mathbb{2}^{2}: \mathcal{A}^{k}(M) \rightarrow$ $\mathcal{A}^{n-k}(M)$. The star operator is an complex-linear isometry and satisfies $* *=$ $(-1)^{k(n-k)}: \mathcal{A}^{k}(M) \rightarrow \mathcal{A}^{k}(M)$. The adjoint $d^{a d}: \mathcal{A}^{k}(M) \rightarrow \mathcal{A}^{k-1}(M)$ of the exterior derivative can be written as $d^{a d}=(-1)^{n(k+1)+1} * d *$.

If $n=2 m$, then one introduces the involution $\tau:=i^{k(k-1)+m} *$ on $k$-forms 9 , p. 574. Then $D_{2 m}=d+d^{a d}$ satisfies $D_{2 m} \tau=-\tau D_{2 m}$. If $\mathcal{A}_{ \pm}^{*}(M)$ denote the \pm 1 eigenbundles of $\tau$, then the operator $D: \mathcal{A}_{+}^{*}(M) \rightarrow \mathcal{A}_{-}^{*}(M)$ is the (even) signature operator. This is an elliptic differential operator of order 1 whose index is the same as the signature of $M$. Clearly, the signature operator is symbolically universal, associated with the $S O(2 m-1)$-equivariant isomorphism of $S O(2 m)$-representations

$$
i(\epsilon-* \epsilon *): \Lambda_{+}^{*}\left(\mathbb{R}^{2 m}\right) \otimes \mathbb{C} \rightarrow \Lambda_{-}^{*}\left(\mathbb{R}^{2 m}\right) \otimes \mathbb{C},
$$

where $\epsilon$ denotes wedge multiplication with the last standard basis vector.

Following 6, we now introduce the odd signature operator on a $2 m$-1-dimensional closed oriented Riemannian manifold $M$. Note that $* *=1$ and $d^{a d}=(-1)^{k} * d *$ : $\mathcal{A}^{k}(M) \rightarrow \mathcal{A}^{k-1}(M)$. The odd signature operator $D=D_{2 m-1}: \bigoplus_{p \geq 0} \mathcal{A}^{2 p}(M) \rightarrow$ $\bigoplus_{p \geq 0} \mathcal{A}^{2 p}(M)$ is defined to be

$$
D_{2 m-1} \phi=i^{m}(-1)^{p+1}(* d-d *) \phi
$$

whenever $\phi \in \mathcal{A}^{2 p}(M)$.

The odd signature operator is universal at the symbolic level, because $d$ and $*$ are universal operators. It is associated with the representation $\Lambda^{e v}\left(\mathbb{R}^{2 m-1}\right)$ and the endomorphism

$$
i^{m-1}(-1)^{p}(* \epsilon-\epsilon *) .
$$

A straightforward, but tedious, calculation shows that

(1) $D$ is formally self-adjoint.

(2) $D^{2}=\Delta=\left(d+d^{a d}\right)^{2}$, the Laplace-Beltrami operator.

Since $\Delta$ is elliptic, it follows that $D$ is elliptic. Moreover, we observe that

$$
\operatorname{ker}(D)=\operatorname{ker}(\Delta)=\bigoplus_{p \geq 0} H^{2 p}(M ; \mathbb{C})
$$

and that consequently

$$
\operatorname{dim} \operatorname{ker} D=\sum_{p \geq 0} \operatorname{dim} H^{2 p}(M ; \mathbb{C}),
$$

which is the main property needed for the proof of Theorem 1.0.2.

\footnotetext{
${ }^{2}$ There might exist different sign conventions about $*$. We are constantly using the definition given in 9 .
} 
4.2. The vanishing theorem. Let $f: E \rightarrow B$ be a smooth oriented $M$-bundle, $M$ a closed oriented $(2 m-1)$-manifold. Assume that we choose a Riemannian metric on the vertical tangent bundle (for any bundle on a paracompact base space such a metric exists; the space of these metrics is contractible). The odd signature operators on the fibres of $f$ fit together to a family of self-adjoint elliptic differential operators. Therefore we have the family index

$$
\operatorname{ind}(D) \in K^{1}(B),
$$

which does not depend on the auxiliary Riemannian metric, but which is an invariant of smooth oriented $M$-bundles. In the universal case, we get an element $\operatorname{ind}(D) \in$ $K^{1}\left(B \operatorname{Diff}^{+}(M)\right)$.

The proof of Theorem 1.0.2 is an immediate consequence of 4.1 .3 and Theorem 4.2 .1 below. Theorem 4.2.1 seems to be well-known to some people working in operator theory, see e.g. [14], 5.1.4. I have included the following rather elmentary proof for the convenience of the reader.

Theorem 4.2.1. Let $B$ be a space and let $A: B \rightarrow \operatorname{Fred}_{s . a}^{0}(H), x \mapsto A_{x}$ be $a$ continuous map such that $x \mapsto \operatorname{dim} \operatorname{ker} A_{x}$ is locally constant. Then $A$ is homotopic to a constant map.

Proof. Step 1: First we show that we can deform $A$ into a family $A^{\prime}$ consisting of invertible operators.

To this end, we note that because the dimension of ker $A_{x}$ is locally constant, the union $K:=\bigcup_{x \in B} \operatorname{ker}\left(A_{x}\right)$ is a (finite-dimensional) vector bundle on $B$. Therefore the projection operator $p_{x}$ onto the kernel of $A_{x}$ depends continuously on $x$ and $p_{x}$ commutes with $A_{x}$ because $A_{x}$ is self-adjoint. Therefore $A_{x}+t p_{x}$ is Fredholm for all $t \in \mathbb{R}$ and $\operatorname{Spec}\left(A_{x}+t p_{x}\right)=\operatorname{Spec} A_{x} \backslash\{0\} \cup\{t\} \subset \mathbb{R}_{\neq 0}$. Thus for $t \neq 0, A_{x}+t p_{x}$ is invertible.

Step 2: By step 1, we assume that $A_{x}$ is invertible for all $x \in B$, in other words $\operatorname{Spec}\left(A_{x}\right) \subset \mathbb{R} \backslash 0$ for all $x \in B$. Let $h: \mathbb{R} \backslash 0 \rightarrow \mathbb{R}$ be the signum function. For any $x$ and any $t \in[0,1]$, the operator

$$
\operatorname{th}(A)+(1-t) A
$$

is a self-adjoint invertible operator (the latter statement is easy to see because $A$ and $h(A)$ commute). For $t=0$, we get $A$ and for $t=1$, we get $h(A)$ which is a self-adjoint involution which is neither essentially positive nor negative.

Step 3: By step 2, we can assume that $A$ is a map from $B$ into the space $\mathcal{P}(H)$ of all involutions $F$ on $H$ such that $\operatorname{Eig}(F, \pm 1)$ are both infinite-dimensional. Let us show that $\mathcal{P}(H)$ is contractible. The unitary group $U(H)$ acts transitively on $\mathcal{P}(H)$ (by conjugation) and the isotropy group at $F$ is $U(\operatorname{Eig}(F, 1)) \times U(\operatorname{Eig}(F,-1))$. Thus we have a continuous bijection

$$
U(H) / U(\operatorname{Eig}(F, 1)) \times U(\operatorname{Eig}(F,-1)) \rightarrow \mathcal{P} .
$$

The map $U(H) \rightarrow \mathcal{P}(H), u \mapsto u F u^{-1}$ has a local section and thus the bijection above is a homeomorphism. The left hand side space is contractible by Kuiper's theorem [21], which completes the proof of the theorem. 
In the proof of the theorem we had the choice between two different contractible spaces of nullhomotopies of $A$; in the first step, we could choose either a positive value or a negative value of the real parameter $t$ (put in another way: the spectral value can be pushed either in the positive or in the negative direction). The concatenation of these two nullhomotopies defines a map $B \rightarrow \Omega \operatorname{Fred}_{\text {s.a. }}^{0} \simeq \Omega U \simeq \mathbb{Z} \times B U$, in other words a class in $K^{0}(B)$. It is not hard to see that this class is the same as the class of the bundle $K \rightarrow B$.

In the case of the odd signature operator, this is the $K$-theory class of the bundle of even cohomology groups. This $K$-theory class is a characteristic class of smooth oriented fibre bundle, nevertheless, it is not induced by an element in $K^{0}(\operatorname{MTSO}(2 m-1))$. This can be seen as follows: certainly there exist odddimensional manifold bundles for which the corresponding class in $K^{0}$ of the base is nonzero (for example, take orientation reversing involutions on $\mathbb{S}^{1}$ and $\mathbb{S}^{2}$. The diagonal action $\mathbb{Z} / 2 \curvearrowright \mathbb{S}^{1} \times \mathbb{S}^{2}$ is then orientation-preserving. Then consider the bundle $\left.E \mathbb{Z} / 2 \times_{\mathbb{Z} / 2}\left(\mathbb{S}^{1} \times b S^{2}\right)\right)$. On the other hand $K_{S O(2 m-1)}^{0}\left(\mathbb{R}^{2 m-1}, \mathbb{R}_{0}^{2 m-1}\right) \cong K_{S O(2 m-1)}^{1+\tau}(*)$ by the Thom isomorphism in twisted $K$-theory ([15]). Here $\tau$ is the twist induced from the central extension $\operatorname{Spin}^{c}(2 m-1) \rightarrow S O(2 m-1)$. On the other hand, $K_{S O(2 m-1)}^{1+\tau}=0$, see [15], p.11. By the Atiyah-Segal completion theorem [8], it follows that $K^{0}(\operatorname{MTSO}(2 m-1)) \cong K^{0}(\operatorname{MNSO}(2 m-1))=\left(K_{S O(2 m-1)}^{0}\left(\mathbb{R}^{2 m-1}, \mathbb{R}_{0}^{2 m-1}\right)\right)^{\wedge}=$ 0 .

4.3. Cohomology calculation. In this section, we indicate how Theorem 1.0 .3 is derived from Theorem 1.0.2. The computation is implicit in [9] and [6] and we shall give only a sketch. First note that the second statement of Theorem 1.0.3 is an immediate consequence of the first one in view of 2.6.1.

Abbreviate $\mathbb{R}_{0}^{n}:=\mathbb{R}^{n} \backslash 0$. Let $\sigma_{2 m-1} \in K_{S O(2 m-1)}^{-1}\left(\mathbb{R}^{2 m-1}, \mathbb{R}_{0}^{2 m-1}\right)$ be the symbol class of the odd signature operator and $\sigma_{2 m} \in K_{S O(2 m)}^{0}\left(\mathbb{R}^{2 m}, \mathbb{R}_{0}^{2 m}\right)$ be the symbol class of the even signature operator. We denote their images in $K^{*}(\operatorname{MNSO}(n))$ by the same symbol. Theorem 1.0 .2 and the index theorem 3.1.1 together yield the identity

$$
\alpha_{E}^{*} \text { th }_{-L_{2 m-1} \otimes \mathbb{C}} \sigma_{2 m-1}=0 \in K^{-1}(B)
$$

for any oriented $2 m-1$-dimensional bundle $p: E \rightarrow B$. To derive 1.0 .3 , we apply the Chern character to the equation 4.3.1.

Proposition 4.3.2. The image of $\sigma_{2 m} \in K_{S O(2 m)}^{0}\left(\mathbb{R}^{2 m}, \mathbb{R}_{0}^{2 m}\right)$ under the restriction homomorphism $K_{S O(2 m)}^{0}\left(\mathbb{R}^{2 m}, \mathbb{R}_{0}^{2 m}\right) \rightarrow K_{S O(2 m-1)}^{0}\left(\left(\mathbb{R}^{2 m-1}, \mathbb{R}_{0}^{2 m-1}\right) \times\left(\mathbb{R}, \mathbb{R}_{0}\right)\right)=$ $K_{S O(2 m-1)}^{1}\left(\mathbb{R}^{2 m-1}, \mathbb{R}_{0}^{2 m-1}\right)$ is the same as $2 \sigma_{2 m-1}$.

Proof. This is contained in the proof of Lemma 4.2 in [6].

Corollary 4.3.3. The element 2 th ${ }_{-L_{2 m-1} \otimes \mathbb{C}} \sigma_{2 m-1} \in K^{1}(\operatorname{MTSO}(2 m-1)) \cong K^{0}\left(\Sigma^{-1} \operatorname{MTSO}(2 m-\right.$ 1)) is the pullback of th ${-L_{2 m} \otimes \mathbb{C}}_{2 m} \in K^{0}(\operatorname{MTSO}(2 m))$ under the spectrum map $\eta: \Sigma^{-1} \operatorname{MTSO}(2 m-1) \rightarrow \operatorname{MTSO}(2 m)$. 
Proof. This follows from 4.3 .2 by the naturality of Thom isomorphism. More precisely, the diagram

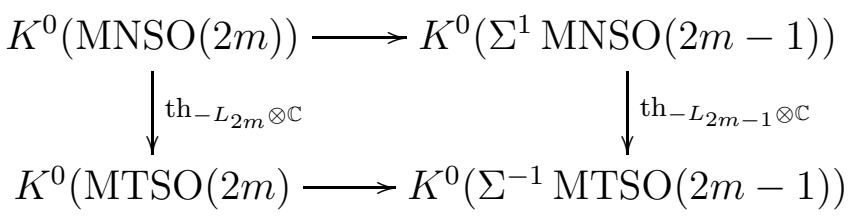

is commutative.

Because the Chern character commutes with suspension homomorphism, Corollary 4.3 .3 implies the identity:

$0=\operatorname{ch}\left(\alpha_{E}^{*} \operatorname{th}_{-L_{2 m-1} \otimes \mathbb{C}} \sigma_{2 m-1}\right)=\alpha_{E}^{*} \operatorname{ch}\left(\operatorname{th}_{-L_{2 m-1} \otimes \mathbb{C}} \sigma_{2 m-1}\right)=\alpha_{E}^{*} \frac{1}{2} \eta^{*} \operatorname{ch}\left(\operatorname{th}_{-L_{2 m} \otimes \mathbb{C}} \sigma_{2 m}\right) \in H^{*}(B ; \mathbb{Q})$.

Recall the calculus of multiplicative sequences [19]. For any power series $q \in \mathbb{Q}^{\times}+$ $x \mathbb{Q}[[x]]$, it associates a cohomology class $\Xi_{U}(q) \in H^{* *}(B U ; \mathbb{Q})=\prod_{n \geq 0} H^{n}(B U ; \mathbb{Q})$. It is multiplicative in the sense that if $\mu: B U \times B U \rightarrow B U$ denotes the Whitney sum map, then $\mu^{*} \Xi_{U}(q)=\Xi_{U}(q) \otimes \Xi_{U}(q)$. Moreover, $\Xi_{U}(q r)=\Xi_{U}(q) \Xi_{U}(r)$. Let $c: B O \rightarrow B U$ be the complexification map; we define $\Xi_{O}(q):=c^{*}\left(\Xi_{U}(q)\right)$.

Let $\operatorname{td}(x)=\frac{x}{1-e^{-x}} \in \mathbb{Q}[[x]]$ and let $\operatorname{Td}:=\Xi_{U}(\operatorname{td}) \in H^{*}(B U ; \mathbb{Q})$ be the Todd class. Similarly, let $h(x):=\frac{x}{2} \operatorname{coth}\left(\frac{x}{2}\right)$ and $\mathcal{L}:=\Xi_{O}(h) \in H^{* *}(B O ; \mathbb{Q})$. This is the Hirzebruch L-class (or rather its modification to be found in [9].

The Chern character ch $: K \rightarrow H \mathbb{Q}$ does not commute with the Thom-isomorphisms in $K$-theory and cohomology. We rather have the formula

$$
\operatorname{chth}_{-V}^{K}(x)=\operatorname{th}_{-V}^{H \mathbb{Q}}(\operatorname{Td}(V) \operatorname{ch}(x)) \in H^{*}(\mathbb{T h}(V) ; \mathbb{Q})
$$

for any complex stable vector bundle $V$. The formula remains correct for the relative Thom isomorphisms; this time we have to interpret the product on the left hand side as multiplication in the module. The Todd class of $L_{2 m} \otimes \mathbb{C}$ is

$$
\operatorname{Td}\left(L_{2 m} \otimes \mathbb{C}\right)=\left.\Xi_{O}\left(\frac{x}{1-e^{-x}} \frac{-x}{1-e^{x}}\right)\right|_{B S O(2 m)}
$$

Therefore

$$
\operatorname{ch}\left(\operatorname{th}_{-L_{2 m} \otimes \mathbb{C}}^{K} \sigma_{2 m}\right)=\operatorname{th}_{-L_{2 m} \otimes \mathbb{C}}^{H \mathbb{Q}}\left(\left.\Xi_{O}\left(\frac{x}{1-e^{-x}} \frac{-x}{1-e^{x}}\right)\right|_{B S O(2 m)} \operatorname{ch}\left(\sigma_{2 m}\right)\right)
$$

It remains to compute $\operatorname{ch}\left(\sigma_{2 m}\right) \in H^{*}(\operatorname{MNSO}(2 m) ; \mathbb{Q})$. The result is

$$
\operatorname{ch}\left(\sigma_{2 m}\right)=\operatorname{th}_{L_{n}}^{H \mathbb{Q}}\left(\Xi_{O}\left(\frac{e^{x}-e^{-x}}{x}\right)\right) .
$$


This is done in 9], p. 576. We leave it to the reader to adapt the argument to our present notation. Putting everything together (and clearing the Thom isomorphisms), we finally obtain:

$0=\frac{1}{2} \alpha_{E}^{*} \eta^{*} \operatorname{th}_{-L_{2 m}}^{H \mathbb{Q}}\left(\left.\Xi_{O}\left(-2 \operatorname{coth}\left(\frac{x}{2}\right)\right)\right|_{B S O(2 m)}\right)=\frac{1}{2} \alpha_{E}^{*} \operatorname{th}_{-L_{2 m-1}}\left(\left.\Xi_{O}\left(-2 \operatorname{coth}\left(\frac{x}{2}\right)\right)\right|_{B S O(2 m-1)}\right)$.

Since in any degree $\left.\left.\left(-2 \operatorname{coth}\left(\frac{x}{2}\right)\right)\right|_{B S O(2 m-1)}\right)$ is a (nonzero) multiple of the Hirzebruch $\mathcal{L}$-class, we conclude that

$$
\alpha_{E}^{*} \operatorname{th}_{-L_{2 m-1}}(\mathcal{L})=0 .
$$

\subsection{Applications of Theorem 1.0.3.}

Proof of Corollary 1.0.4: Recall the power series expansion

$$
x / 2 \operatorname{coth}(x / 2)=1+\sum_{k=1}^{\infty}(-1)^{k+1} \frac{B_{k}}{(2 k) !} x^{2 k}
$$

and recall that $B_{k}$ is a nonzero rational number. On the other hand $H^{*}(B S O(3))=$ $\mathbb{Q}\left[p_{1}\right]$ and therefore $\mathcal{L}=1+\sum_{k=1}^{\infty}(-1)^{k+1} \frac{B_{k}}{(2 k) !} p_{1}^{k}$. Thus the components of $\mathcal{L}$ form an additive basis of $H^{*}(B S O(3))$. Therefore, by Theorem 1.0.3, $H^{*}(\operatorname{MTSO}(3) ; \mathbb{Q}) \rightarrow$ $H^{*}\left(\Sigma^{\infty}\left(B \operatorname{Diff}^{+}(M)\right)_{+} ; \mathbb{Q}\right)$ is trivial. By 2.7.1, this finishes the proof.

Corollary 4.4.1. Let $E \rightarrow B$ be an oriented fibre bundle over a closed oriented manifold with odd-dimensional closed fibres. Then $\operatorname{sign}(E)=0$.

This is an old theorem, which was first mentioned in [5] (without proof), proven in [26] and later, as a special case of a much more sophisticated theory, in [22]. Moreover, we will show below that Corollary 4.4.1implies Theorem 1.0.3 by standard methods. However, our Theorem 1.0.2 from which we derived 1.0.3 is much stronger.

Proof of Corollary 4.4.1. Because $T E \cong f^{*} T B \oplus T_{v} E$, we have

$$
\operatorname{sign}(E)=\langle\mathcal{L}(T E) ;[E]\rangle=\left\langle\mathcal{L}\left(f^{*} T B\right) \mathcal{L}\left(T_{v} E\right) ;[E]\right\rangle=\left\langle\mathcal{L}(T B) f_{!}\left(\mathcal{L}\left(T_{v} E\right)\right) ;[B]\right\rangle .
$$

By Theorem 1.0.3, $f_{!}\left(\mathcal{L}\left(T_{v} E\right)\right)=0$.

To derive 1.0.3 from 4.4.1, observe first that $H_{*}\left(B \operatorname{Diff}^{+}(M) ; \mathbb{Q}\right) \cong \Omega_{*}^{f r}\left(B \operatorname{Diff}^{+}(M)\right) \otimes$ $\mathbb{Q}$ (the framed bordism group) by Pontrjagin's theorem and Serre's finiteness theorem. Therefore, to show that $\alpha_{M}^{*}$ th $\mathcal{L}=0$, it suffices to show that $h^{*} \alpha_{M}^{*}$ th $\mathcal{L}=0$ whenever $h: B \rightarrow B \operatorname{Diff}^{+}(M)$ is a map with $B$ a framed manifold, classifying an $M$-bundle $f: E \rightarrow B$. If $B$ is framed, then $\mathcal{L}(T B)=1$ and therefore by 4.4 .2 and 4.4 .1

$$
0=\operatorname{sign}(E)=\langle\mathcal{L}(T E) ;[E]\rangle=\left\langle f_{!}\left(\mathcal{L}\left(T_{v} E\right)\right) \mathcal{L}(T B) ;[B]\right\rangle=\left\langle f_{!}\left(\mathcal{L}\left(T_{v} E\right)\right) ;[B]\right\rangle .
$$

Therefore 1.0 .3 follows. 


\section{NONVANishing RESUlt FOR Other GENERALIZED MMM-ClASSES}

Let $\Phi \in H^{4 m}(B S O ; \mathbb{Q})$ be a polynomial in the Pontrjagin classes. We can ask whether for $2 n$-1-dimensional oriented manifold bundles $f: E \rightarrow B$ the generalized MMM-class $f_{!}\left(\Phi\left(T_{v} E\right)\right) \in H^{4 m-2 n+1}(B ; \mathbb{Q})$ vanishes (for multiples of the Hirzebruch $\mathcal{L}$-class, we have seen that these classes always vanish). We have a partial answer for $n=2 m$, i.e. for bundles of $4 m-1$-dimensional manifolds.

Theorem 5.0.4. For any $\Phi \in H^{4 m}(B S O ; \mathbb{Q})$ which is not a multiple of the Hirzebruch class $\mathcal{L}_{m}$, there exists a $4 m-1$-dimensional closed oriented manifold $M$ and a smooth oriented $M$-bundle $f: E \rightarrow \mathbb{S}^{1}$ such that the generalized MMM-class $f_{!}\left(\Phi\left(T_{v} M_{g}\right)\right) \in H^{1}\left(\mathbb{S}^{1} ; \mathbb{Q}\right)$ is nontrivial.

Proof. By Thom's computation of the rational oriented cobordism ring $\Omega_{*}^{S O} \otimes \mathbb{Q} \cong$ $\mathbb{Q}\left[\left[\mathbb{C P}^{2}\right],\left[\mathbb{C P}^{4}\right],\left[\mathbb{C P}^{6}\right], \ldots\right]$ and its description via characteristic numbers [28], we can find an element $x \in \Omega_{4 m}^{S O} \otimes \mathbb{Q}$ such that $\operatorname{sign}(x)=\mathcal{L}(x)=0$ and $\Phi(x) \neq 0$ (Here we identify a polynomial in the Pontrjagin classes with the bordism invariant it induces). After multiplication by a nonzero integer we find an actual manifold $N$ with these properties. By the result of Neumann [32], $N$ is oriented cobordant to a manifold $E$ which admits a fibre bundle $f: E \rightarrow \mathbb{S}^{1}$. Take $M$ to be the fibre of $f$; the bundle $f$ is clearly an oriented smooth $M$-bundle. The manifold $E$ has the same characteristic numbers as $N$. The little computation

$$
\left\langle f_{!}\left(\Phi\left(T_{v} E\right) ;\left[\mathbb{S}^{1}\right]\right\rangle=\left\langle\Phi\left(T_{v} E ;[E]\right\rangle=\langle\Phi(T E) ;[E]\rangle \neq 0\right.\right.
$$

finishes the proof.

The proof of Neumann's result is highly nonconstructive and we do not know a single concrete example. We can use 5.0.4 to construct oriented manifold bundles with nonvanishing generalized MMM-classes of higher order.

Let $\mu: B O \times B O \rightarrow B O$ be the addition map. Let $\Phi \in H^{* *}(B O ; \mathbb{Q})=$ $\prod_{i=0}^{\infty} H^{i}(B O ; \mathbb{Q})$ be a multiplicative sequence, i.e. $\mu^{*} \Phi=\Phi \times \Phi$. If $f_{i}: E_{i} \rightarrow B_{i}$, $i=1, \ldots, r$ are oriented manifold bundles, the generalized MMM-classes of the product $f=f_{1} \times f_{2} \ldots \times f_{r}: E=E_{1} \times \ldots E_{r} \rightarrow B=B_{1} \times \ldots B_{r}$ are given by

$$
f_{!}\left(\Phi\left(T_{v} E\right)\right)=\bigotimes_{i=1}^{r}\left(f_{i}\right)_{!} \Phi\left(T_{v} E_{i}\right) \in H^{*}(B) .
$$

Let $B_{i}=\mathbb{S}^{1}$, let $m_{1}, \ldots m_{r}$ be such that $\Phi_{m_{i}} \in H^{4 m_{i}}(B O ; \mathbb{Q})$ is not a multiple of the Hirzebruch $\mathcal{L}$-class and let $f_{i}: E_{i} \rightarrow \mathbb{S}^{1}$ be bundles of fibre dimension $4 m_{i}$ as in Theorem 5.0.4. Then the degree $r$ part of the generalized MMM-class of the product $f_{!}\left(\Phi\left(T_{v} E\right)\right) \in H^{r}\left(\left(\mathbb{S}^{1}\right)^{r} ; \mathbb{Q}\right) \cong \mathbb{Q}$ is nontrivial. This gives a lot of examples of nonzero generalized MMM-classes.

\section{A REAL REFINEMENT AND THE ONE-DIMENSIONAL CASE}

Recall the formula for the odd signature operator: $D=i^{m}(-1)^{p+1}(* d-d *)$ on a $2 m-1$-dimensional manifold. If $m$ is odd $(2 m-1=1,5, \ldots)$, then $-i D$ is a real, skew-adjoint operator, acting on real-valued differential forms. As such, it has an 
index in $K O^{-1}(B)$ (again, $f: E \rightarrow B$ is a smooth oriented fibre bundle of fibre dimension $2 m-1)$, compare [11].

The question we consider now is whether this refined index is also trivial. We have to check whether the argument in the proof of Theorem 4.2.1 goes through with $-i D$ instead of $D$ in the space of real, skew-adjoint Fredholm operators. It turns out that step 2 can be changed appropriately (we deform an invertible operator into one with $F^{2}=-1$ ). The argument for step 3 can be applied to the space of skew-adjoint real Fredholm operators $F$ with $F^{2}=-1$, because Kuipers theorem is true for the isometry group of a real Hilbert space as well. The problem is with step 1.

Let $H \rightarrow B$ be the finite-dimensional real vector bundle formed out of the kernels of the real odd signature operator. Recall that the fibre of $H$ at $x \in B$ is the vector space $\bigoplus_{p \geq 0} H^{2 p}\left(f^{-1}(x) ; \mathbb{R}\right)$.

In order to make sense out of the deformation in step 1 , it is not enough to know that $H$ is a real vector bundle, but also that $H$ admits a skew-adjoint invertible endomorphism. Such an endomorphism is, up to homotopy, the same as a complex structure on $H$.

Therefore:

Theorem 6.0.5. Let $f: E \rightarrow B$ be an oriented smooth $M$-bundle, $M$ of dimension $4 r+1$. Then the real family index of the odd signature operator $\operatorname{ind}_{\mathbb{R}} D \in K O^{-1}(B)$ is trivial if and only if the real vector bundle $H \rightarrow B$ admits a complex structure.

The first obstruction to find a complex structure on $H$ is $\operatorname{dim} H(\bmod 2)$. This agrees with the Kervaire semi-characteristic $\operatorname{Kerv}(M)$. More generally, we can interpret this result in terms of the exact sequence

$$
K^{-2}(B) \stackrel{\gamma}{\rightarrow} K O^{0}(B) \stackrel{\delta}{\rightarrow} K O^{-1}(B)
$$

compare e.g. [20], Thm 5.18. The map $\gamma$ is the inverse to the Bott map, composed with the realification map $K^{0} \rightarrow K O^{0}$ and $\delta$ is the product with the generator of $K O^{-1}(*) \cong \mathbb{Z} / 2$. The image $\delta([H]) \in K O^{-1}(B)$ agrees with the real index.

It is worth to study the 1-dimensional case explicitly. The Madsen-Tillmann spectrum is $\operatorname{MTSO}(1) \cong \Sigma^{-1} \Sigma^{\infty} \mathbb{S}^{0}$. It is well-known that Diff ${ }^{+}\left(\mathbb{S}^{1}\right) \simeq \mathbb{S}^{1}$; therefore $B \operatorname{Diff}^{+}\left(\mathbb{S}^{1}\right) \simeq \mathbb{C P} \mathbb{P}^{\infty}$. The Madsen-Tillmann map $\alpha: \Sigma^{\infty} \mathbb{C P}_{+}^{\infty} \rightarrow \Sigma^{-1} \Sigma^{\infty} \mathbb{S}^{0}$ can be identified with the circle transfer. The restriction $\Sigma^{\infty} \mathbb{S}^{0} \rightarrow \Sigma^{-1} \Sigma^{\infty} \mathbb{S}^{0}$ of $\alpha$ to the basepoint is simply the generator $\eta \in \pi_{1}\left(\Sigma^{\infty} \mathbb{S}^{0}\right) \cong \mathbb{Z} / 2$.

The odd signature operator on $\mathbb{S}^{1}$ is simply $D=-i * d$ on $C^{\infty}\left(\mathbb{S}^{1}\right)$. If $\mathbb{S}^{1}$ has a Riemannian metric with volume $a$ and $x$ is a coordinate $\mathbb{S}^{1} \rightarrow \mathbb{R} / a \mathbb{Z}$ preserving orientation and length, then $D=-i \frac{d}{d x}$. The symbol is $\operatorname{smb}_{D}(d x)=-1$. Using this, it is easy to see that $\sigma_{1} \in K_{S O(1)}^{-1}\left(\mathbb{R}, \mathbb{R}_{0}\right)=K^{0}\left(\mathbb{R}^{2}, \mathbb{R}_{0}^{2}\right)$ is the Bott class. Thus the universal symbol is a generator of $K^{-1}(\operatorname{MTSO}(1)) \cong \mathbb{Z}$.

The vanishing theorem 1.0 .2 in this case can be obtained much easier because $K^{-1}\left(\mathbb{C P}^{\infty}\right)=0$. In fact, the vanishing theorem for the topological index follows immediately from this fact, without any use of elliptic operator theory. 
On the other hand, the Kervaire semi-characteristic of $\mathbb{S}^{1}$ is clearly nonzero and therefore the real index of the signature operator is nonzero; it is a generator of $K O^{-1}\left(\mathbb{C P}^{\infty}\right) \cong \mathbb{Z} / 2$ (the latter isomorphism follows easily from the main result of [4]). The restriction of the real index to the basepoint is the generator of $\mathrm{KO}^{-1}(*)=$ $\mathbb{Z} / 2$. These things check with the fact that $\eta \in \pi_{1}\left(\Sigma^{\infty} \mathbb{S}^{0}\right)$ cannot be detected by complex $K$-theory, but by real $K$-theory.

\section{VANISHING THEOREMS IN MOD $p$ COHOMOLOGY AND AN OPEN PROBLEM}

We have seen that for any oriented closed 3-manifold $M$, the map $\alpha_{E_{M}}: B \operatorname{Diff}^{+}(M) \rightarrow$ $\operatorname{MTSO}(3)$ is trivial in rational cohomology. Moreover, we learnt that for each $r$, the Madsen-Tillmann map of an oriented $\mathbb{S}^{4 r+1}$-bundle is nontrivial (because $\left.\operatorname{Kerv}\left(\mathbb{S}^{4 r+1}\right)=1\right)$. Furthermore, for any $r \geq 2$, there exists an $4 r-1$-dimensional manifold $M$ and and oriented $M$-bundle $E \rightarrow \mathbb{S}^{1}$ such that $\alpha_{E}^{*}$ is nontrivial in rational cohomology. What we do not know is whether there exists an oriented closed 3-manifold $M$ such that $\alpha_{E_{M}}: B \operatorname{Diff}^{+}(M) \rightarrow \Omega^{\infty} \operatorname{MTSO}(3)$ is nontrivial in homology.

In this section, we sketch two methods to derive from 1.0.2 that $\alpha_{E_{M}}^{*}: H^{4 k-3}\left(\operatorname{MTSO}(3) ; \mathbb{F}_{p}\right) \rightarrow$ $H^{4 k-3}\left(B \operatorname{Diff}^{+}(M) ; \mathbb{F}_{p}\right)$ vanishes for certain values of $k$ and primes $p$.

Theorem 7.0.6. For any oriented closed 3-manifold $M$ and for any $k \geq 1$, the map $\alpha_{E_{M}}^{*}: H^{4 k-3}\left(\operatorname{MTSO}(3) ; \mathbb{F}_{p}\right) \rightarrow H^{4 k-3}\left(B \operatorname{Diff}^{+}(M) ; \mathbb{F}_{p}\right)$ is trivial for all primes $p$ with $p \geq 2 k$ and $p$ not dividing the numerator of $B_{k}$ (these are almost all primes, for a fixed $k$ ).

Theorem 7.0.7. For any oriented closed 3-manifold $M$ and for any odd prime $p$, the map $\alpha_{E_{M}}^{*}: H^{4 k+1}\left(\operatorname{MTSO}(3) ; \mathbb{F}_{p}\right) \rightarrow H^{4 k+1}\left(B \operatorname{Diff}^{+}(M) ; \mathbb{F}_{p}\right)$ is trivial when $k=\frac{1}{2}(p-1) i$ for some $i \in \mathbb{N}$.

Note that neither of the sets of pairs $(k, p)$ provided by the two theorems contains the other one. Also, they do not exhaust all values of $(k, p)$. Neither theorem makes a statement about the prime 2 . The methods of the proof of both theorem can be used to derive vanishing theorems for all odd dimensions (and the method of 7.0.7 gives a result about the prime 2 as well), but here we confine ourselves to the case of dimension 3.

Proof of Theorem 7.0.6: The symbol of the odd signature operator $\operatorname{th}(\sigma) \in K^{-1}(\mathrm{MTSO}(3))$, when considered as a map $\operatorname{MTSO}(3) \rightarrow \Sigma^{-1} K$, can be lifted to connective $K$-theory, i.e. to a map

$$
\kappa: \operatorname{MTSO}(3) \rightarrow \Sigma^{-3} \mathbf{k}
$$

The composition $\kappa \circ \alpha: B \operatorname{Diff}^{+}(M)_{+} \rightarrow \operatorname{MTSO}(3) \rightarrow \Sigma^{-3} \mathbf{k}$ is still nullhomotopic. The point of the proof is a theorem of Adams [2], [1] about the spectrum cohomology of $\mathbf{k}$. In general, the class $s_{r}:=r ! \mathrm{ch}_{r} \in H^{2 r}(B U ; \mathbb{Z})$ is not a spectrum cohomology class, i.e. it does not lie in the image of the cohomology suspension $H^{2 r}(\mathbf{k} ; \mathbb{Z}) \rightarrow H^{2 r}(B U ; \mathbb{Z})$. The result of [2], [1] is that a certain multiple $m(r) \mathrm{ch}_{r}$ actually is a spectrum cohomology class. The number $m(r)$ is given by 
$m(r):=\prod_{p} p^{\left[\frac{r}{p-1}\right]}$. The product goes over all prime numbers and for $x \in \mathbb{R},[x]$ is the largest integer which is less or equal than $x$ (thus, it involves only primes $p$ with $p-1 \leq r)$. Moreover, $u_{r}:=m(r) \operatorname{ch}_{r}$ is a generator of $H^{2 r}(\mathbf{k} ; \mathbb{Z}) \cong \mathbb{Z}, r \geq 0$ (all other cohomology groups of $\mathbf{k}$ are trivial). If $p$ is an odd prime then $H^{*}(\operatorname{MTSO}(3) ; \mathbb{Z})$ has no $p$-torsion and so $H^{*}(\operatorname{MTSO}(3) ; \mathbb{Z}) \otimes \mathbb{F}_{p} \cong H^{*}\left(\operatorname{MTSO}(3) ; \mathbb{F}_{p}\right)$. Therefore, if $\kappa^{*}\left(\Sigma^{-3} u_{r}\right) \in H^{2 r-3}(\operatorname{MTSO}(3))$ reduces to a generator of $H^{2 r-3}\left(\operatorname{MTSO}(3) ; \mathbb{F}_{p}\right)$, then $\alpha: H^{2 r-3}\left(\operatorname{MTSO}(3) ; \mathbb{F}_{p}\right) \rightarrow H^{2 r-3}\left(B \operatorname{Diff}^{+}(M) ; \mathbb{F}_{p}\right)$ is the zero map. Up to powers of 2 which we can disregard since $p$ is assumed to be odd, $\kappa^{*}$ maps $\Sigma^{-3} u_{2 r} \in H^{2 r-3}\left(\Sigma^{-3} \mathbf{k}\right)$ to

$$
\pm \frac{B_{r}}{(2 r) !} m(2 r)\left(u_{-3} p_{1}^{r}\right)
$$

(it is not hard to derive that this class is integral from Von Staudt's theorem and Lemma 2.1. of 33 ). This reduces to a generator mod $p$ if $p$ does not divide $\frac{B_{r}}{(2 r) !} m(2 r)$ which is certainly the case if $p \geq 2 r$ and $p$ does not divide the numerator of $B_{r}$.

Proof of Theorem 7.0.7: Look at the diagram:

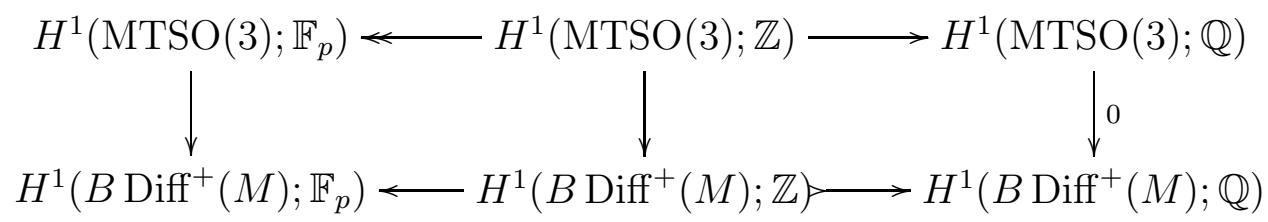

The right-hand vertical arrow is zero by Corollary 1.0.4 and the lower right horizontal arrow is injective $\left(H^{1}(X ; \mathbb{Z}) \rightarrow H^{1}(X ; \mathbb{Q})\right.$ is injective for any space $\left.X\right)$. The upper left horizontal arrow is surjective since $p$ is odd. An easy diagram chase shows that $H^{1}\left(\operatorname{MTSO}(3) ; \mathbb{F}_{p}\right) \rightarrow H^{1}\left(B \operatorname{Diff}^{+}(M) ; \mathbb{F}_{p}\right)$ is also trivial.

Let $\mathcal{A}_{p}$ denote the mod $p$-Steenrod algebra. Then the restriction of $\alpha$ to $\mathcal{A}_{p}$. $H^{1}(\operatorname{MTSO}(3))$ is trivial because $\alpha$ is a spectrum map. Let $x:=p_{1} \in H^{4}\left(B S O(3) ; \mathbb{F}_{4}\right)$ be the first Pontrjagin class and let $\mathcal{P}$ be the total Steenrod power operation. We will compute $\mathcal{P}\left(\operatorname{th}_{-L_{3}}(x)\right) \in H^{*}\left(\operatorname{MTSO}(3) ; \mathbb{F}_{p}\right)$. This is done using a formula by $\mathrm{Wu}$ (compare [28, Thm 19.7). Let $r=\frac{1}{2}(p-1), \mathcal{P}^{i}$ has degree 4ri. Wu's formula states, in the present context, that

$$
\mathcal{P}\left(\operatorname{th}_{-L_{3}} x\right)=\operatorname{th}_{-L_{3}}\left(\left(x+x^{p}\right)\left(1+x^{r}\right)^{-1}\right) .
$$

Therefore $\mathcal{P}^{i}\left(\operatorname{th}_{-L_{3}} x\right) \in H^{1+4 r i}\left(\operatorname{MTSO}(3) ; \mathbb{F}_{p}\right)$ agrees with $\operatorname{th}_{-L_{3}}\left(x^{r i+1}\right)$, multiplied by the coefficient of $z^{r i+1}$ in the power series

$$
\left(z+z^{p}\right)\left(1+z^{r}\right)^{-1}=\sum_{l \geq 0}(-1)^{l}\left(z^{r l+1}+z^{r l+p}\right) .
$$

It is clear that this coefficient is a unit in $\mathbb{F}_{p}^{\times}$. Thus $\mathcal{P}^{i}\left(\operatorname{th}_{-L_{3}} x\right) \in H^{1+4 r i}\left(\operatorname{MTSO}(3) ; \mathbb{F}_{p}\right)$ is a generator and we have argued above that $\alpha^{*} \mathcal{P}^{i}\left(\operatorname{th}_{-L_{3}} x\right)=0$. This concludes the proof. 
It appears to be quite difficult to find an example of a 3-manifold such that $\alpha$ : $\Sigma^{\infty} B \operatorname{Diff}^{+}(M)_{+} \rightarrow \operatorname{MTSO}(3)$ is nonzero in cohomology. Many computations which will not be reproduced here suggest that $\alpha$ could very well vanish in cohomology with arbitrary coefficients. However:

Proposition 7.0.9. For $M=\mathbb{S}^{3}$, the Madsen-Tillmann map $\Sigma^{\infty} B S O(4)_{+} \rightarrow$ $\operatorname{MTSO}(3)$ of the universal $\mathbb{S}^{3}$-bundle is not nullhomotopic.

Sketch of Proof (The author owes this argument to O. Randal-Wiliams): Recall that $\alpha$ fits into the cofibre sequence 2.4.1.

$$
\Sigma^{\infty} \mathrm{BSO}(4)_{+} \stackrel{\alpha}{\rightarrow} \operatorname{MTSO}(3) \stackrel{\eta}{\rightarrow} \Sigma \mathrm{MTSO}(4)
$$

If $\alpha$ were nullhomotopic, then there exists a splitting $s: \Sigma \operatorname{MTSO}(4) \rightarrow \operatorname{MTSO}(3)$ of $\eta$ (i.e. $s \circ \eta=\mathrm{id}$ ). In the sequel we assume that the map

$$
\eta^{*}: H^{*}\left(\operatorname{MTSO}(4) ; \mathbb{F}_{3}\right) \rightarrow H^{*}\left(\Sigma^{-1} \operatorname{MTSO}(3) ; \mathbb{F}_{3}\right)
$$

has a right inverse $s^{*}$ as a map of $\mathcal{A}_{3}$-modules and show that this is impossible. Let $u_{-3}$ be the Thom class of $-L_{3}$ and we write $u_{-3} \cdot x$ for th $L_{-L_{3}}(x)$ (recall that this is a module structure); similarly for $\operatorname{MTSO}(4)$. Since $\eta^{*} u_{-4}=\Sigma^{-1} u_{3}$, it follows that $s^{*} \Sigma^{-1} u_{-3}=u_{-4}$ and thus that $Q u_{-4}=\Sigma^{-1} s^{*} Q_{u_{3}}$ for any $Q \in \mathcal{A}_{3}$. Put $Q=\mathcal{P}^{3}+\mathcal{P}^{1} \mathcal{P}^{2}$. Using the formulae

$$
\mathcal{P}^{1} p_{1}=p_{1}^{2} ; \mathcal{P}^{2} p_{1}=p_{1}^{3} ; \mathcal{P}^{1} p_{1}^{2}=-p_{1}\left(p_{1}^{2}+p_{2}\right) ; \mathcal{P}^{1} p_{2}=p_{1} p_{2}
$$

for the Steenrod operations on $B S O(4)$ (and hence, by putting $p_{2}=0$, also on $B S O(3))$ and the formula

$$
\mathcal{P}\left(u_{4}\right)=u_{-4}\left(K\left(p_{1}, p_{2}\right)\right)
$$

where $K$ is the multiplicative sequence associated with $(1+x)^{-1}$, we get

$$
\left(\mathcal{P}^{3}+\mathcal{P}^{1} \mathcal{P}^{2}\right)\left(u_{-4}\right)=p_{1} p_{2}
$$

on $\operatorname{MTSO}(4)$. This is a contradiction since $\left(\mathcal{P}^{3}+\mathcal{P}^{1} \mathcal{P}^{2}\right)\left(u_{-3}\right)=0$ by the above formula.

We conclude this paper by asking the question:

Question 7.0.10. Does there exist an oriented closed 3-manifold $M$ and a prime $p$, such that $\alpha_{E_{M}}^{*}: \tilde{H}^{*}\left(\operatorname{MTSO}(3) ; \mathbb{F}_{p}\right) \rightarrow \tilde{H}^{*}\left(B \operatorname{Diff}^{+}(M) ; \mathbb{F}_{p}\right)$ is nontrivial? 


\section{REFERENCES}

[1] J. F. Adams: Chern characters revisited. Illinois Journal of Mathematics 17 (1973), 333336.

[2] J. F. Adams: On Chern characters and the structure of the unitary groups. Proceed. of the Cambridge Philosophical Society 57 (1961), 189-199.

[3] J. F. Adams: Stable homotopy and generalised homology. Reprint of the 1974 original. Chicago Lectures in Mathematics. University of Chicago Press, Chicago, (1995).

[4] D. W. Anderson: The real K-Theory of classifying spaces. Proc. National Acad. Sci. U.S.A. 51 (1964), 634636.

[5] M. F. Atiyah: The signature of fibre bundles. Global Analysis (Papers in Honor of K. Kodaira), pp. 73-84. Univ. Tokyo Press, Tokyo (1969).

[6] M. F. Atiyah; V. K. Patodi; I. M. Singer: Spectral asymmetry and Riemannian geometry III, Math. Proc. Cambridge Philos. Soc 79 (1976), 71-99.

[7] M. F. Atiyah; G. Segal: Twisted K-theory. Ukr. Mat. Visn. 1 (2004), 287-330.

[8] M. F. Atiyah, G. Segal: Equivariant K-Theory and completion. J. Differential Geometry 3 (1969).

[9] M. F. Atiyah; I. M. Singer: The index of elliptic operators III. Ann. of Math. 87 (1968), 546-604.

[10] M. F. Atiyah; I. M. Singer: The index of elliptic operators IV. Ann. of Math. 93 (1971), 119-138.

[11] M. F. Atiyah; I. M. Singer: The index of elliptic operators V. Ann. of Math. 93 (1971), 139-149.

[12] M. F. Atiyah; I. M. Singer: Index theory for skew-adjoint Fredholm operators. Publ. Math. IHES 37 (1969), 5-26.

[13] J. C. Becker, D. H. Gottlieb: The transfer map and fiber bundles. Topology 14 (1975), $1-12$.

[14] U. Bunke: Index Theory, Eta Forms, and Deligne Cohomology. Memoirs of the American Mathematical Society 198 (2009).

[15] D. Freed, M. Hopkins, C. Teleman, Loop groups and twisted K-theory I. Preprint, arXiv:0711.1906, 2007.

[16] S. Galatius: Mod 2 homology of the stable spin mapping class group. Math. Ann. 334 (2006), 439-455.

[17] S. Galatius; I. Madsen; U. Tillmann; M. Weiss: The homotopy type of the cobordism category. Preprint arXiv.org/math.AT/0605249, (2006), to appear in Acta Mathematica.

[18] A. Hatcher; N. Wahl: Stabilization for mapping class groups of 3-manifolds. Preprint, ArXiv:math/0709.2173 (2007).

[19] F. Hirzebruch: Neue topologische Methoden in der algebraischen Geometrie. SpringerVerlag, (1962).

[20] M. Karoubi: K-Theory. An Introduction. Reprint of the 1978 edition, Springer-Verlag 2008.

[21] N. H. Kuiper: The homotopy type of the unitary group of Hilbert space. Topology 3 (1965), 19-30.

[22] W. Lück; A. Ranicki: Surgery obstructions in fiber bundles. J. Pure Appl. Algebra 81 (1992), 139-189.

[23] H. B. Lawson; M. Michelsohn: Spin geometry. Princeton University Press, (1989).

[24] I. Madsen; U. Tillmann: The stable mapping class group and $\mathbb{C P}_{-1}^{\infty}$. Invent. Math. 145 (2001), 509-544.

[25] I. Madsen; M. Weiss: The stable moduli space of Riemann surfaces: Mumford's conjecture. Ann. of Math. 165 (2007), 843-941.

[26] W. Meyer: Die Signatur von Faserbündeln und lokalen Koeffizientensystemen. Bonner Mathematische Schriften 53 (1972).

[27] J. W. Milnor; J. C. Moore: On the structure of Hopf algebras. Ann. of Math. 81 (1965), 211-264. 
[28] J. W. Milnor; J. Stasheff, J.: Characteristic classes. Annals of Mathematics Studies, No. 76. Princeton University Press, Princeton, N. J. (1974).

[29] E. Y. Miller: The homology of the mapping class group. J. Differential Geom. 24 (1986), $1-14$.

[30] S. Morita: Characteristic classes of surface bundles. Invent. Math. 90 (1987), 551-577.

[31] D. Mumford: Towards an enumerative geometry of the moduli space of curves. Arithmetic and geometry, Vol. II, 271-328, Progr. Math., 36, Birkhüser Boston, Boston, MA, 1983.

[32] Neumann, W. D.: Fibering over the circle within a bordism class. Math. Ann 192 (1971), 191-192.

[33] G. Pappas: Integral Riemann-Roch theorem. Invent. Math. 170 (2007), 455-481.

[34] B. L. Reinhart: Cobordism and the Euler number. Topology 2 (1963) 173-177.

[35] Y. B. Rudyak: On Thom spectra, orientability and cobordism. Springer-Verlag, 1998.

[36] U. Tillmann: On the homotopy of the stable mapping class group. Invent. Math. 130 (1997), 257-275.

Mathematisches Institut der Universität Bonn, Beringstrasse 1, 53115 Bonn, BundesRepublik Deutschland

E-mail address: ebert@math.uni-bonn.de 